\title{
Overview of Activity Coefficient of Thiophene at Infinite Dilution in Ionic Liquids and their Modelling Using COSMO-RS
}

Pranesh Matheswaran, ${ }^{1,2}$ Cecilia Devi Wilfred, ${ }^{1,2 *}$ Kiki A. Kurnia, ${ }^{1,3}$ Anita Binti Ramli. ${ }^{2}$

${ }^{1}$ Centre of Research in Ionic Liquids (CORIL), ${ }^{2}$ Department of Fundamental and Applied Science, and ${ }^{3}$ Department of Chemical Engineering, Universiti Teknologi Petronas, Bandar Seri Iskandar, Perak 32610 Malaysia.

\section{SUPPORTING INFORMATION}


Table 1S. Name and abbreviation of ions composing the ionic liquids in which the data of activity coefficient of water at infinite dilution is available

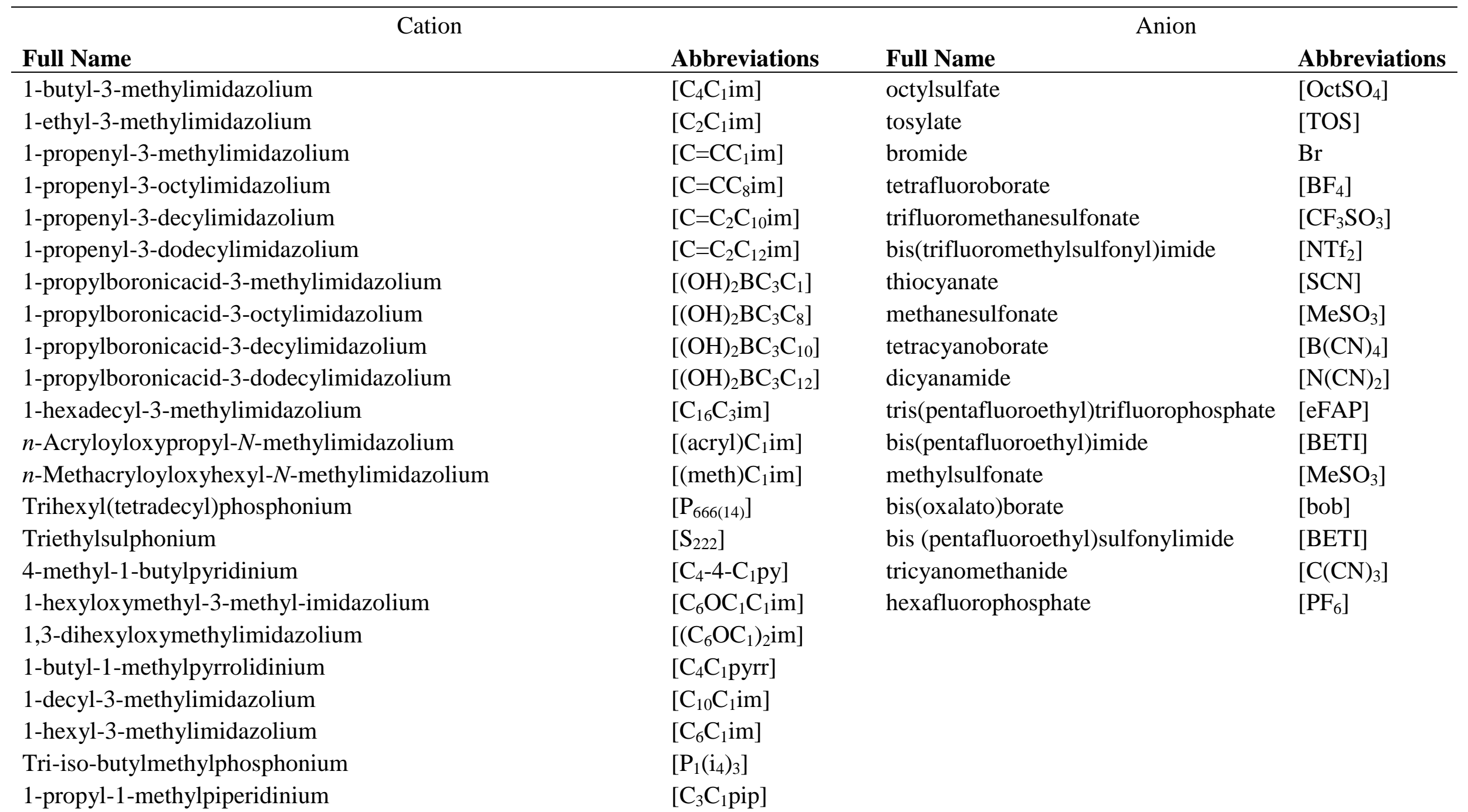


3-methyl-1-butylpyridinium

1-(3-Hydroxypropyl)pyridinium

1-(2-Hydroxyethyl)-3-methylimidazolium

1-methylethylether-3-methylimidazolium

1,3- dimethoxyimidazolium

1-(3-Cyanopropyl)-3-methylimidazolium

1-butyl-1-methylpiperidinium

1-hexyl-3-methylpyridinium

$\mathrm{N}$-octylisoquinolinium

1,3-didecyl-2-methylimidazolium

1-hexyl-1-methylpyrrolidinium

1-octyl-1-methylpyrrolidinium

1-decyl-1-methylpyrrolidinium

1-(2-methoxyethyl)-1-methylpiperidinium

4-(2-methoxyethyl)-4-methylmorpholinium

1-(2-methoxyethyl)-1-methylpyrrolidinium

1,3,4,6, 7,8-hexahydro-1-methyl-2H-pyrimido[1,2-a]pyrimidine $\mathrm{N}$-hexylisoquinolinium

1-propyl-1-methylpyrrolidinium

1-pentyl-1-methylpiperidinium

1-hexyl-1-methylpiperidinium

1-butyl-1-methylmorpholinium

1-butyl-4-methylpyridinium
$\left[\mathrm{C}_{4}-3-\mathrm{C}_{1} \mathrm{py}\right]$

[HOC 3 py]

[ $\mathrm{HOC}_{2} \mathrm{C}_{1} \mathrm{im}$ ]

[ $\mathrm{COC}_{2} \mathrm{C}_{1} \mathrm{im}$ ]

[(CO $\left.)_{2} \mathrm{im}\right]$

[NC-C ${ }_{3} \mathrm{C}_{1} \mathrm{im}$ ]

$\left[\mathrm{C}_{4} \mathrm{C}_{1}\right.$ pip]

[C $\left.\mathrm{C}_{6}-3-\mathrm{C}_{1} \mathrm{py}\right]$

[C $\mathrm{C}_{8}$-iQui]

[ $\left.\mathrm{C}_{10} \mathrm{C}_{10} \mathrm{C}_{1} \mathrm{im}\right]$

[ $\mathrm{C}_{6} \mathrm{C}_{1}$ pyrr]

$\left[\mathrm{C}_{8} \mathrm{C}_{1}\right.$ pyrr]

$\left[\mathrm{C}_{10} \mathrm{C}_{1}\right.$ pyrr]

[ $\mathrm{COC}_{2} \mathrm{C}_{1}$ pip]

[ $\mathrm{COC}_{2} \mathrm{C}_{1}$ morp]

[ $\mathrm{COC}_{2} \mathrm{C}_{1}$ pyrr]

[MTBDH]

[C $\mathrm{C}_{6}$-iQui]

[ $\mathrm{C}_{3} \mathrm{C}_{1}$ pyrr]

[ $\mathrm{C}_{5} \mathrm{C}_{1}$ pip]

$\left[\mathrm{C}_{6} \mathrm{C}_{1}\right.$ pip]

[C $\mathrm{C}_{4} \mathrm{C}_{1}$ morp]

[C $\left.\mathrm{C}_{4}-4-\mathrm{C}_{1} \mathrm{py}\right]$ 
Table 2S. Experiment, $\gamma_{s}^{\infty}$,exp and predicted, $\gamma_{s}^{\infty}$, COSMO-RS activity coefficient of thiophene at infinite dilution in IL using COSMO-RS along with the absolute average deviation, AAD\% calculated using equation $3 \mathrm{~S}$

\begin{tabular}{|c|c|c|c|c|c|}
\hline Ref & Ionic Liquids & $\mathbf{T} / \mathbf{K}$ & $\gamma^{\infty, \exp }$ & $\gamma^{\infty, C O S M O-R S}$ & ARD \% \\
\hline 1 & [C4C1im][OctSO4] & 323.15 & 0.73 & 0.800 & 9.6 \\
\hline 1 & [C4C1im][OctSO4] & 333.15 & 0.78 & 0.784 & 0.6 \\
\hline 1 & [C4C1im][OctSO4] & 343.15 & 0.82 & 0.769 & 6.2 \\
\hline 1 & {$[\mathrm{C} 2 \mathrm{C} 1 \mathrm{im}][\mathrm{TOS}]$} & 323.15 & 1.41 & 1.189 & 15.6 \\
\hline 2 & {$[\mathrm{C}=\mathrm{CC} 1 \mathrm{im}] \mathrm{Br}$} & 323.15 & 1.48 & 0.902 & 39.1 \\
\hline 2 & {$[\mathrm{C}=\mathrm{CC} 8 \mathrm{im}] \mathrm{Br}$} & 323.15 & 0.93 & 0.806 & 13.4 \\
\hline 3 & [C4C1im][CF3SO3] & 298.15 & 1.14 & 1.257 & 10.2 \\
\hline 3 & [C4C1im][CF3SO3] & 308.15 & 1.15 & 1.226 & 6.6 \\
\hline 3 & [C4C1im][CF3SO3] & 318.15 & 1.15 & 1.195 & 3.9 \\
\hline 3 & [C4C1im][CF3SO3] & 328.15 & 1.16 & 1.164 & 0.4 \\
\hline 3 & [C4C1im][CF3SO3] & 338.15 & 1.17 & 1.134 & 3.0 \\
\hline 3 & [C4C1im][CF3SO3] & 348.15 & 1.18 & 1.105 & 6.3 \\
\hline 3 & [C4C1im][CF3SO3] & 358.15 & 1.18 & 1.077 & 8.7 \\
\hline 3 & [C4C1im][CF3SO3] & 368.15 & 1.19 & 1.051 & 11.7 \\
\hline 4 & {$[($ acryl $) \mathrm{Clim}] \mathrm{Br}$} & 313.15 & 2.47 & 1.115 & 54.9 \\
\hline 4 & {$[($ acryl $) \mathrm{C} 1 \mathrm{im}] \mathrm{Br}$} & 323.15 & 2.68 & 1.146 & 57.2 \\
\hline 4 & {$[($ meth $) \mathrm{C} 1 \mathrm{im}] \mathrm{Br}$} & 313.15 & 1.5 & 0.858 & 42.8 \\
\hline 5 & {$[\mathrm{P} 666(14)][\mathrm{NTf} 2]$} & 303.15 & 0.42 & 0.373 & 11.1 \\
\hline 5 & [P666(14)][NTf2] & 323.15 & 0.4 & 0.373 & 6.7 \\
\hline 5 & {$[\mathrm{P} 666(14)][\mathrm{NTf} 2]$} & 343.15 & 0.38 & 0.373 & 1.8 \\
\hline 6 & {$[\mathrm{C} 4 \mathrm{C} 1 \mathrm{im}][\mathrm{SCN}]$} & 298.15 & 1.214 & 1.790 & 47.4 \\
\hline 6 & {$[\mathrm{C} 4 \mathrm{C} 1 \mathrm{im}][\mathrm{SCN}]$} & 308.15 & 1.241 & 1.746 & 40.7 \\
\hline 6 & {$[\mathrm{C} 4 \mathrm{C} 1 \mathrm{im}][\mathrm{SCN}]$} & 318.15 & 1.256 & 1.702 & 35.5 \\
\hline 6 & {$[\mathrm{C} 4 \mathrm{C} 1 \mathrm{im}][\mathrm{SCN}]$} & 328.15 & 1.269 & 1.657 & 30.6 \\
\hline 6 & {$[\mathrm{C} 4 \mathrm{C} 1 \mathrm{im}][\mathrm{SCN}]$} & 338.15 & 1.294 & 1.613 & 24.6 \\
\hline 6 & {$[\mathrm{C} 4 \mathrm{C} 1 \mathrm{im}][\mathrm{SCN}]$} & 348.15 & 1.317 & 1.569 & 19.1 \\
\hline 6 & {$[\mathrm{C} 4 \mathrm{C} 1 \mathrm{im}][\mathrm{SCN}]$} & 358.15 & 1.331 & 1.527 & 14.7 \\
\hline 6 & {$[\mathrm{C} 4 \mathrm{C} 1 \mathrm{im}][\mathrm{SCN}]$} & 368.15 & 1.361 & 1.486 & 9.2 \\
\hline 7 & [S222][NTf2] & 298.15 & 0.926 & 0.750 & 19.0 \\
\hline
\end{tabular}




\begin{tabular}{|c|c|c|c|c|c|}
\hline 7 & [S222][NTf2] & 308.15 & 0.935 & 0.735 & 21.4 \\
\hline 7 & [S222][NTf2] & 318.15 & 0.943 & 0.722 & 23.5 \\
\hline 7 & [S222][NTf2] & 328.15 & 0.951 & 0.708 & 25.5 \\
\hline 7 & [S222][NTf2] & 338.15 & 0.958 & 0.696 & 27.4 \\
\hline 7 & [S222][NTf2] & 348.15 & 0.965 & 0.684 & 29.1 \\
\hline 7 & [S222][NTf2] & 358.15 & 0.971 & 0.673 & 30.7 \\
\hline 7 & [S222][NTf2] & 368.15 & 0.977 & 0.662 & 32.3 \\
\hline 8 & [C4-4-C1py][NTf2] & 298.15 & 0.609 & 0.631 & 3.6 \\
\hline 8 & [C4-4-C1py][NTf2] & 308.15 & 0.624 & 0.622 & 0.4 \\
\hline 8 & [C4-4-C1py][NTf2] & 318.15 & 0.637 & 0.613 & 3.8 \\
\hline 8 & [C4-4-C1py][NTf2] & 328.15 & 0.649 & 0.604 & 6.9 \\
\hline 8 & [C4-4-C1py][NTf2] & 338.15 & 0.661 & 0.596 & 9.9 \\
\hline 8 & [C4-4-C1py][NTf2] & 348.15 & 0.672 & 0.588 & 12.5 \\
\hline 8 & [C4-4-C1py][NTf2] & 358.15 & 0.683 & 0.581 & 15.0 \\
\hline 8 & [C4-4-C1py][NTf2] & 368.15 & 0.694 & 0.574 & 17.4 \\
\hline 9 & [C6OC1C1im][NTf2] & 298.15 & 0.743 & 0.702 & 5.5 \\
\hline 9 & [C6OC1C1im][NTf2] & 308.15 & 0.744 & 0.684 & 8.1 \\
\hline 9 & [C6OC1C1im][NTf2] & 318.15 & 0.744 & 0.667 & 10.3 \\
\hline 9 & [C6OC1C1im][NTf2] & 328.15 & 0.745 & 0.651 & 12.6 \\
\hline 9 & [C6OC1C1im][NTf2] & 338.15 & 0.746 & 0.637 & 14.6 \\
\hline 9 & [C6OC1C1im][NTf2] & 348.15 & 0.746 & 0.624 & 16.4 \\
\hline 9 & [C6OC1C1im][NTf2] & 358.15 & 0.747 & 0.611 & 18.2 \\
\hline 9 & [C6OC1C1im][NTf2] & 368.15 & 0.747 & 0.600 & 19.7 \\
\hline 9 & {$[(\mathrm{C} 6 \mathrm{OC} 1) 2 \mathrm{im}][\mathrm{NTf} 2]$} & 298.15 & 0.578 & 0.553 & 4.4 \\
\hline 9 & {$[(\mathrm{C} 6 \mathrm{OC} 1) 2 \mathrm{im}][\mathrm{NTf} 2]$} & 308.15 & 0.575 & 0.541 & 5.9 \\
\hline 9 & [(C6OC1)2im][NTf2] & 318.15 & 0.571 & 0.531 & 7.1 \\
\hline 9 & {$[(\mathrm{C} 6 \mathrm{OC} 1) 2 \mathrm{im}][\mathrm{NTf} 2]$} & 328.15 & 0.568 & 0.521 & 8.3 \\
\hline 9 & {$[(\mathrm{C} 6 \mathrm{OC} 1) 2 \mathrm{im}][\mathrm{NTf} 2]$} & 338.15 & 0.565 & 0.512 & 9.4 \\
\hline 9 & {$[(\mathrm{C} 6 \mathrm{OC} 1) 2 \mathrm{im}][\mathrm{NTf} 2]$} & 348.15 & 0.562 & 0.504 & 10.4 \\
\hline 9 & {$[(\mathrm{C} 6 \mathrm{OC} 1) 2 \mathrm{im}][\mathrm{NTf} 2]$} & 358.15 & 0.559 & 0.496 & 11.2 \\
\hline 9 & {$[(\mathrm{C} 6 \mathrm{OC} 1) 2 \mathrm{im}][\mathrm{NTf} 2]$} & 368.15 & 0.557 & 0.489 & 12.2 \\
\hline 10 & [C4C1pyrr][CF3SO3] & 298.15 & 1.02 & 0.877 & 14.0 \\
\hline 10 & [C4C1pyrr][CF3SO3] & 308.15 & 1.04 & 0.871 & 16.2 \\
\hline
\end{tabular}




\begin{tabular}{|c|c|c|c|c|c|}
\hline 10 & [C4C1pyrr][CF3SO3] & 318.15 & 1.06 & 0.864 & 18.5 \\
\hline 10 & [C4C1pyrr][CF3SO3] & 328.15 & 1.08 & 0.857 & 20.7 \\
\hline 10 & [C4C1pyrr][CF3SO3] & 338.15 & 1.1 & 0.848 & 22.9 \\
\hline 10 & [C4C1pyrr][CF3SO3] & 348.15 & 1.11 & 0.840 & 24.4 \\
\hline 10 & [C4C1pyrr][CF3SO3] & 358.15 & 1.13 & 0.831 & 26.5 \\
\hline 10 & [C4C1pyrr][CF3SO3] & 368.15 & 1.14 & 0.821 & 28.0 \\
\hline 11 & [C4C1im][BF4] & 303.15 & 1.56 & 1.673 & 7.3 \\
\hline 11 & [C4C1im][BF4] & 313.15 & 1.52 & 1.633 & 7.5 \\
\hline 11 & {$[\mathrm{C} 4 \mathrm{C} 1 \mathrm{im}][\mathrm{BF} 4]$} & 323.15 & 1.54 & 1.592 & 3.4 \\
\hline 11 & [C4C1im][BF4] & 333.15 & 1.49 & 1.551 & 4.1 \\
\hline 12 & {$[\mathrm{C} 2 \mathrm{C} 1 \mathrm{im}][\mathrm{MeSO} 3]$} & 318.15 & 2.04 & 0.986 & 51.7 \\
\hline 12 & {$[\mathrm{C} 2 \mathrm{C} 1 \mathrm{im}][\mathrm{MeSO} 3]$} & 323.15 & 2.06 & 1.003 & 51.3 \\
\hline 12 & [C2C1im][MeSO3] & 333.15 & 2.11 & 1.034 & 51.0 \\
\hline 12 & {$[\mathrm{C} 2 \mathrm{C} 1 \mathrm{im}][\mathrm{MeSO} 3]$} & 343.15 & 2.16 & 1.060 & 50.9 \\
\hline 12 & {$[\mathrm{C} 2 \mathrm{C} 1 \mathrm{im}][\mathrm{MeSO} 3]$} & 353.15 & 2.2 & 1.083 & 50.8 \\
\hline 13 & [C4-4-C1py][SCN] & 298.15 & 0.963 & 1.241 & 28.9 \\
\hline 13 & [C4-4-C1py][SCN] & 308.15 & 0.984 & 1.225 & 24.5 \\
\hline 13 & [C4-4-C1py][SCN] & 318.15 & 1 & 1.208 & 20.8 \\
\hline 13 & {$[\mathrm{C} 4-4-\mathrm{C} 1 \mathrm{py}][\mathrm{SCN}]$} & 328.15 & 1.02 & 1.189 & 16.6 \\
\hline 13 & [C4-4-C1py][SCN] & 338.15 & 1.03 & 1.170 & 13.6 \\
\hline 13 & {$[\mathrm{C} 4-4-\mathrm{C} 1 \mathrm{py}][\mathrm{SCN}]$} & 348.15 & 1.05 & 1.151 & 9.6 \\
\hline 13 & [C4-4-C1py][SCN] & 358.15 & 1.07 & 1.132 & 5.8 \\
\hline 13 & [C4-4-C1py][SCN] & 368.15 & 1.08 & 1.113 & 3.0 \\
\hline 13 & {$[\mathrm{C} 4 \mathrm{C} 1 \mathrm{pyrr}][\mathrm{SCN}]$} & 318.15 & 1.07 & 0.983 & 8.2 \\
\hline 13 & {$[\mathrm{C} 4 \mathrm{C} 1 \mathrm{pyrr}][\mathrm{SCN}]$} & 328.15 & 1.11 & 0.983 & 11.5 \\
\hline 13 & {$[\mathrm{C} 4 \mathrm{C} 1$ pyrr $][\mathrm{SCN}]$} & 338.15 & 1.15 & 0.981 & 14.7 \\
\hline 13 & {$[\mathrm{C} 4 \mathrm{C} 1 \mathrm{pyrr}][\mathrm{SCN}]$} & 348.15 & 1.19 & 0.978 & 17.8 \\
\hline 13 & {$[\mathrm{C} 4 \mathrm{C} 1 \mathrm{pyrr}][\mathrm{SCN}]$} & 358.15 & 1.23 & 0.974 & 20.8 \\
\hline 14 & [C4C1im][TOS] & 338.15 & 1.11 & 0.964 & 13.1 \\
\hline 14 & {$[\mathrm{C} 4 \mathrm{C} 1 \mathrm{im}][\mathrm{TOS}]$} & 348.15 & 1.13 & 0.950 & 15.9 \\
\hline 14 & {$[\mathrm{C} 4 \mathrm{C} 1 \mathrm{im}][\mathrm{TOS}]$} & 358.15 & 1.14 & 0.936 & 17.9 \\
\hline 14 & {$[\mathrm{C} 4 \mathrm{C} 1 \mathrm{im}][\mathrm{TOS}]$} & 368.15 & 1.16 & 0.921 & 20.6 \\
\hline 15 & {$[\mathrm{C} 10 \mathrm{C} 1 \mathrm{im}][\mathrm{B}(\mathrm{CN}) 4]$} & 328.15 & 0.527 & 0.609 & 15.5 \\
\hline
\end{tabular}




\begin{tabular}{|c|c|c|c|c|c|}
\hline 15 & {$[\mathrm{C} 10 \mathrm{C} 1 \mathrm{im}][\mathrm{B}(\mathrm{CN}) 4]$} & 338.15 & 0.536 & 0.592 & 10.5 \\
\hline 15 & {$[\mathrm{C} 10 \mathrm{C} 1 \mathrm{im}][\mathrm{B}(\mathrm{CN}) 4]$} & 348.15 & 0.543 & 0.578 & 6.4 \\
\hline 15 & {$[\mathrm{C} 10 \mathrm{C} 1 \mathrm{im}][\mathrm{B}(\mathrm{CN}) 4]$} & 358.15 & 0.552 & 0.564 & 2.3 \\
\hline 15 & {$[\mathrm{C} 10 \mathrm{C} 1 \mathrm{im}][\mathrm{B}(\mathrm{CN}) 4]$} & 368.15 & 0.561 & 0.553 & 1.5 \\
\hline 16 & {$[\mathrm{C} 6 \mathrm{C} 1 \mathrm{im}][\mathrm{SCN}]$} & 298.15 & 1.24 & 1.450 & 17.0 \\
\hline 16 & {$[\mathrm{C} 6 \mathrm{C} 1 \mathrm{im}][\mathrm{SCN}]$} & 308.15 & 1.28 & 1.405 & 9.7 \\
\hline 16 & {$[\mathrm{C} 6 \mathrm{C} 1 \mathrm{im}][\mathrm{SCN}]$} & 318.15 & 1.31 & 1.360 & 3.9 \\
\hline 16 & {$[\mathrm{C} 6 \mathrm{C} 1 \mathrm{im}][\mathrm{SCN}]$} & 328.15 & 1.34 & 1.318 & 1.6 \\
\hline 16 & {$[\mathrm{C} 6 \mathrm{C} 1 \mathrm{im}][\mathrm{SCN}]$} & 338.15 & 1.37 & 1.278 & 6.7 \\
\hline 16 & {$[\mathrm{C} 6 \mathrm{C} 1 \mathrm{im}][\mathrm{SCN}]$} & 348.15 & 1.4 & 1.239 & 11.5 \\
\hline 16 & {$[\mathrm{C} 6 \mathrm{C} 1 \mathrm{im}][\mathrm{SCN}]$} & 358.15 & 1.43 & 1.203 & 15.9 \\
\hline 16 & {$[\mathrm{C} 6 \mathrm{C} 1 \mathrm{im}][\mathrm{SCN}]$} & 368.15 & 1.45 & 1.169 & 19.4 \\
\hline 17 & [P1(i4)3][TOS] & 298.15 & 0.901 & 0.477 & 47.0 \\
\hline 17 & [P1(i4)3][TOS] & 308.15 & 0.848 & 0.482 & 43.2 \\
\hline 17 & [P1(i4)3][TOS] & 318.15 & 0.843 & 0.485 & 42.4 \\
\hline 17 & [P1(i4)3][TOS] & 328.15 & 0.818 & 0.488 & 40.3 \\
\hline 17 & [P1(i4)3][TOS] & 338.15 & 0.812 & 0.491 & 39.5 \\
\hline 17 & [P1(i4)3][TOS] & 348.15 & 0.805 & 0.493 & 38.7 \\
\hline 17 & [P1(i4)3][TOS] & 358.15 & 0.804 & 0.495 & 38.4 \\
\hline 18 & [C3C1pip][NTf2] & 308.15 & 0.809 & 0.595 & 26.4 \\
\hline 18 & [C3C1pip][NTf2] & 318.15 & 0.819 & 0.590 & 28.0 \\
\hline 18 & [C3C1pip][NTf2] & 328.15 & 0.825 & 0.584 & 29.2 \\
\hline 18 & [C3C1pip][NTf2] & 338.15 & 0.838 & 0.579 & 30.9 \\
\hline 18 & [C3C1pip][NTf2] & 348.15 & 0.844 & 0.573 & 32.1 \\
\hline 18 & [C3C1pip][NTf2] & 358.15 & 0.858 & 0.568 & 33.8 \\
\hline 19 & [C4-3-C1py][CF3SO3] & 318.15 & 0.898 & 0.911 & 1.4 \\
\hline 19 & [C4-3-C1py][CF3SO3] & 328.15 & 0.919 & 0.897 & 2.4 \\
\hline 19 & [C4-3-C1py][CF3SO3] & 338.15 & 0.937 & 0.883 & 5.8 \\
\hline 19 & [C4-3-C1py][CF3SO3] & 348.15 & 0.955 & 0.869 & 9.0 \\
\hline 19 & [C4-3-C1py][CF3SO3] & 358.15 & 0.972 & 0.855 & 12.0 \\
\hline 20 & [HOC3py][eFAP] & 308.15 & 0.888 & 0.521 & 41.3 \\
\hline 20 & [HOC3py][eFAP] & 318.15 & 0.898 & 0.516 & 42.5 \\
\hline 20 & [HOC3py][eFAP] & 328.15 & 0.908 & 0.511 & 43.7 \\
\hline
\end{tabular}




\begin{tabular}{|c|c|c|c|c|c|}
\hline 20 & [HOC3py][eFAP] & 338.15 & 0.918 & 0.506 & 44.9 \\
\hline 20 & [HOC3py][eFAP] & 348.15 & 0.927 & 0.501 & 46.0 \\
\hline 20 & {$[\mathrm{HOC} 3 \mathrm{py}][\mathrm{eFAP}]$} & 358.15 & 0.936 & 0.497 & 46.9 \\
\hline 21 & [HOC2C1im][NTf2] & 323.15 & 1.77 & 1.526 & 13.8 \\
\hline 21 & [HOC2C1im][NTf2] & 333.15 & 1.77 & 1.446 & 18.3 \\
\hline 21 & {$[\mathrm{HOC} 2 \mathrm{C} 1 \mathrm{im}][\mathrm{NTf} 2]$} & 343.15 & 1.71 & 1.373 & 19.7 \\
\hline 21 & {$[\mathrm{HOC} 2 \mathrm{C} 1 \mathrm{im}][\mathrm{NTf} 2]$} & 298.15 & 1.86 & 1.759 & 5.4 \\
\hline 21 & [COC2C1im][NTf2] & 333.15 & 1.01 & 0.934 & 7.5 \\
\hline 21 & {$[\mathrm{COC} 2 \mathrm{C} 1 \mathrm{im}][\mathrm{NTf} 2]$} & 343.15 & 1.02 & 0.840 & 17.6 \\
\hline 21 & {$[\mathrm{COC} 2 \mathrm{C} 1 \mathrm{im}][\mathrm{NTf} 2]$} & 353.15 & 1.01 & 0.817 & 19.1 \\
\hline 21 & [COC2C1im][NTf2] & 298.15 & 1.01 & 0.796 & 21.2 \\
\hline 21 & {$[(\mathrm{CO}) 2 \mathrm{im}][\mathrm{NTf} 2]$} & 313.15 & 1.83 & 1.168 & 36.2 \\
\hline 21 & {$[(\mathrm{CO}) 2 \mathrm{im}][\mathrm{NTf} 2]$} & 323.15 & 1.8 & 1.119 & 37.8 \\
\hline 21 & {$[(\mathrm{CO}) 2 \mathrm{im}][\mathrm{NTf} 2]$} & 333.15 & 1.76 & 1.074 & 39.0 \\
\hline 22 & {$[\mathrm{C} 4 \mathrm{C} 1 \mathrm{pyrr}][\mathrm{N}(\mathrm{CN}) 2]$} & 318.15 & 0.851 & 0.870 & 2.2 \\
\hline 22 & {$[\mathrm{C} 4 \mathrm{C} 1 \mathrm{pyrr}][\mathrm{N}(\mathrm{CN}) 2]$} & 323.15 & 0.868 & 0.866 & 0.2 \\
\hline 22 & {$[\mathrm{C} 4 \mathrm{C} 1 \mathrm{pyrr}][\mathrm{N}(\mathrm{CN}) 2]$} & 333.15 & 0.901 & 0.858 & 4.8 \\
\hline 22 & {$[\mathrm{C} 4 \mathrm{C} 1 \mathrm{pyrr}][\mathrm{N}(\mathrm{CN}) 2]$} & 343.15 & 0.931 & 0.850 & 8.7 \\
\hline 22 & {$[\mathrm{C} 4 \mathrm{C} 1 \mathrm{pyrr}][\mathrm{N}(\mathrm{CN}) 2]$} & 353.15 & 0.962 & 0.841 & 12.6 \\
\hline 23 & {$[\mathrm{C} 2 \mathrm{C} 1 \mathrm{im}][\mathrm{B}(\mathrm{CN}) 4]$} & 328.15 & 0.966 & 1.929 & 99.7 \\
\hline 23 & {$[\mathrm{C} 2 \mathrm{C} 1 \mathrm{im}][\mathrm{B}(\mathrm{CN}) 4]$} & 338.15 & 0.976 & 1.810 & 85.5 \\
\hline 23 & {$[\mathrm{C} 2 \mathrm{C} 1 \mathrm{im}][\mathrm{B}(\mathrm{CN}) 4]$} & 348.15 & 0.983 & 1.704 & 73.4 \\
\hline 23 & {$[\mathrm{C} 2 \mathrm{C} 1 \mathrm{im}][\mathrm{B}(\mathrm{CN}) 4]$} & 358.15 & 0.992 & 1.609 & 62.2 \\
\hline 24 & {$[\mathrm{C} 4 \mathrm{C} 1 \mathrm{pip}][\mathrm{SCN}]$} & 318.15 & 1.1 & 0.860 & 21.8 \\
\hline 24 & {$[\mathrm{C} 4 \mathrm{C} 1 \mathrm{pip}][\mathrm{SCN}]$} & 328.15 & 1.13 & 0.860 & 23.9 \\
\hline 24 & {$[\mathrm{C} 4 \mathrm{C} 1 \mathrm{pip}][\mathrm{SCN}]$} & 338.15 & 1.17 & 0.859 & 26.6 \\
\hline 24 & {$[\mathrm{C} 4 \mathrm{C} 1 \mathrm{pip}][\mathrm{SCN}]$} & 348.15 & 1.2 & 0.857 & 28.6 \\
\hline 24 & {$[\mathrm{C} 4 \mathrm{C} 1 \mathrm{pip}][\mathrm{SCN}]$} & 358.15 & 1.23 & 0.853 & 30.6 \\
\hline 25 & [C6-3-C1py][TOS] & 338.15 & 0.753 & 0.693 & 8.0 \\
\hline 25 & [C6-3-C1py][TOS] & 348.15 & 0.765 & 0.687 & 10.2 \\
\hline 25 & [C6-3-C1py][TOS] & 358.15 & 0.776 & 0.681 & 12.2 \\
\hline 25 & [C6-3-C1py][TOS] & 368.15 & 0.787 & 0.675 & 14.2 \\
\hline 26 & {$[\mathrm{C} 4 \mathrm{C} 1 \mathrm{pyrr}][\mathrm{B}(\mathrm{CN}) 4]$} & 318.15 & 0.659 & 0.638 & 3.3 \\
\hline
\end{tabular}




\begin{tabular}{|c|c|c|c|c|}
\hline 26 & {$[\mathrm{C} 4 \mathrm{C} 1 \mathrm{pyrr}][\mathrm{B}(\mathrm{CN}) 4]$} & 328.15 & 0.663 & 0.629 \\
\hline 26 & {$[\mathrm{C} 4 \mathrm{C} 1 \mathrm{pyrr}][\mathrm{B}(\mathrm{CN}) 4]$} & 338.15 & 0.684 & 0.620 \\
\hline 26 & {$[\mathrm{C} 4 \mathrm{C} 1 \mathrm{pyrr}][\mathrm{B}(\mathrm{CN}) 4]$} & 348.15 & 0.702 & 0.612 \\
\hline 26 & {$[\mathrm{C} 4 \mathrm{C} 1 \mathrm{pyrr}][\mathrm{B}(\mathrm{CN}) 4]$} & 358.15 & 0.711 & 0.604 \\
\hline 26 & {$[\mathrm{C} 4 \mathrm{C} 1 \mathrm{pyrr}][\mathrm{B}(\mathrm{CN}) 4]$} & 368.15 & 0.734 & 0.597 \\
\hline 27 & [C8-iQui][NTf2] & 328.15 & 0.564 & 0.474 \\
\hline 27 & [C8-iQui][NTf2] & 338.15 & 0.571 & 0.470 \\
\hline 27 & [C8-iQui][NTf2] & 348.15 & 0.575 & 0.467 \\
\hline 27 & [C8-iQui][NTf2] & 358.15 & 0.58 & 0.463 \\
\hline 27 & [C8-iQui][NTf2] & 368.15 & 0.586 & 0.460 \\
\hline 28 & [HOC3py][NTf2] & 318.15 & 1.27 & 1.301 \\
\hline 28 & [HOC3py][NTf2] & 328.15 & 1.28 & 1.241 \\
\hline 28 & [HOC3py][NTf2] & 338.15 & 1.29 & 1.185 \\
\hline 28 & [HOC3py][NTf2] & 348.15 & 1.29 & 1.133 \\
\hline 28 & [HOC3py][NTf2] & 358.15 & 1.3 & 1.086 \\
\hline 28 & [HOC3py][NTf2] & 368.15 & 1.31 & 1.043 \\
\hline 28 & [HOC3py][NTf2] & 378.15 & 1.31 & 1.003 \\
\hline 29 & [C6C1im][eFAP] & 313.15 & 0.577 & 0.363 \\
\hline 29 & [C6C1im][eFAP] & 333.15 & 0.602 & 0.369 \\
\hline 29 & [C6C1im][eFAP] & 353.15 & 0.604 & 0.374 \\
\hline 29 & {$[\mathrm{C} 4 \mathrm{C} 1 \mathrm{im}][\mathrm{BETI}]$} & 323.15 & 0.977 & 0.556 \\
\hline 29 & [C4C1im][BETI] & 343.15 & 0.973 & 0.541 \\
\hline 29 & {$[\mathrm{C} 4 \mathrm{C} 1 \mathrm{im}][\mathrm{BETI}]$} & 363.15 & 0.981 & 0.528 \\
\hline 29 & {$[\mathrm{C} 10 \mathrm{C} 10 \mathrm{C} 1 \mathrm{im}][\mathrm{NTf} 2]$} & 323.15 & 0.422 & 0.391 \\
\hline 29 & {$[\mathrm{C} 10 \mathrm{C} 10 \mathrm{C} 1 \mathrm{im}][\mathrm{NTf} 2]$} & 333.15 & 0.423 & 0.390 \\
\hline 29 & {$[\mathrm{C} 10 \mathrm{C} 10 \mathrm{C} 1 \mathrm{im}][\mathrm{NTf} 2]$} & 343.15 & 0.424 & 0.390 \\
\hline 30 & [C6C1pyrr][NTf2] & 323.15 & 0.7 & 0.520 \\
\hline 30 & [C6C1pyrr][NTf2] & 333.15 & 0.71 & 0.517 \\
\hline 30 & [C6C1pyrr][NTf2] & 343.15 & 0.72 & 0.513 \\
\hline 30 & [C8C1pyrr][NTf2] & 323.15 & 0.57 & 0.479 \\
\hline 30 & [C8C1pyrr][NTf2] & 333.15 & 0.55 & 0.477 \\
\hline 30 & [C8C1pyrr][NTf2] & 343.15 & 0.53 & 0.475 \\
\hline 30 & [C10C1pyrr][NTf2] & 323.15 & 0.6 & 0.453 \\
\hline
\end{tabular}




\begin{tabular}{|c|c|c|c|c|c|}
\hline 30 & [C10C1pyrr][NTf2] & 333.15 & 0.6 & 0.451 & 24.9 \\
\hline 30 & [C10C1pyrr][NTf2] & 343.15 & 0.61 & 0.449 & 26.3 \\
\hline 31 & {$[\mathrm{C} 2 \mathrm{C} 1 \mathrm{im}][\mathrm{MeSO} 3]$} & 308.15 & 1.87 & 0.948 & 49.3 \\
\hline 32 & [C4C1pyrr][eFAP] & 318.15 & 0.635 & 0.381 & 39.9 \\
\hline 32 & [C4C1pyrr][eFAP] & 328.15 & 0.646 & 0.384 & 40.6 \\
\hline 32 & [C4C1pyrr][eFAP] & 338.15 & 0.659 & 0.386 & 41.4 \\
\hline 32 & [C4C1pyrr][eFAP] & 348.15 & 0.671 & 0.389 & 42.1 \\
\hline 32 & [C4C1pyrr][eFAP] & 358.15 & 0.686 & 0.391 & 43.0 \\
\hline 32 & [C4C1pyrr][eFAP] & 368.15 & 0.696 & 0.393 & 43.6 \\
\hline 33 & {$[\mathrm{C} 6 \mathrm{C} 1 \mathrm{im}][\mathrm{B}(\mathrm{CN}) 4]$} & 318.15 & 0.636 & 0.904 & 42.2 \\
\hline 33 & {$[\mathrm{C} 6 \mathrm{C} 1 \mathrm{im}][\mathrm{B}(\mathrm{CN}) 4]$} & 328.15 & 0.647 & 0.868 & 34.1 \\
\hline 33 & {$[\mathrm{C} 6 \mathrm{C} 1 \mathrm{im}][\mathrm{B}(\mathrm{CN}) 4]$} & 338.15 & 0.657 & 0.835 & 27.1 \\
\hline 33 & {$[\mathrm{C} 6 \mathrm{C} 1 \mathrm{im}][\mathrm{B}(\mathrm{CN}) 4]$} & 348.15 & 0.666 & 0.806 & 21.0 \\
\hline 33 & {$[\mathrm{C} 6 \mathrm{C} 1 \mathrm{im}][\mathrm{B}(\mathrm{CN}) 4]$} & 358.15 & 0.678 & 0.780 & 15.0 \\
\hline 33 & {$[\mathrm{C} 6 \mathrm{C} 1 \mathrm{im}][\mathrm{B}(\mathrm{CN}) 4]$} & 368.15 & 0.689 & 0.756 & 9.7 \\
\hline 34 & [COC2C1pip][NTf2] & 318.15 & 0.787 & 0.727 & 7.6 \\
\hline 34 & [COC2C1pip][NTf2] & 328.15 & 0.798 & 0.712 & 10.7 \\
\hline 34 & [COC2C1pip][NTf2] & 338.15 & 0.808 & 0.698 & 13.6 \\
\hline 34 & [COC2C1pip][NTf2] & 348.15 & 0.819 & 0.685 & 16.3 \\
\hline 34 & [COC2C1pip][NTf2] & 358.15 & 0.829 & 0.673 & 18.9 \\
\hline 34 & [COC2C1pip][NTf2] & 368.15 & 0.839 & 0.661 & 21.2 \\
\hline 35 & [COC2C1morp][NTf2] & 318.15 & 1.09 & 1.090 & 0.0 \\
\hline 35 & [COC2C1morp][NTf2] & 328.15 & 1.08 & 1.047 & 3.1 \\
\hline 35 & [COC2C1morp][NTf2] & 338.15 & 1.08 & 1.007 & 6.7 \\
\hline 35 & {$[\mathrm{COC} 2 \mathrm{C} 1 \mathrm{morp}][\mathrm{NTf} 2]$} & 348.15 & 1.08 & 0.971 & 10.1 \\
\hline 35 & [COC2C1morp][NTf2] & 358.15 & 1.08 & 0.938 & 13.2 \\
\hline 35 & [COC2C1morp][NTf2] & 368.15 & 1.08 & 0.907 & 16.0 \\
\hline 36 & [COC2C1pyrr][NTf2] & 318.15 & 0.885 & 0.690 & 22.0 \\
\hline 36 & [COC2C1pyrr][NTf2] & 328.15 & 0.897 & 0.679 & 24.3 \\
\hline 36 & [COC2C1pyrr][NTf2] & 338.15 & 0.909 & 0.669 & 26.5 \\
\hline 36 & [COC2C1pyrr][NTf2] & 348.15 & 0.918 & 0.658 & 28.3 \\
\hline 36 & [COC2C1pyrr][NTf2] & 358.15 & 0.929 & 0.648 & 30.2 \\
\hline 36 & [COC2C1pyrr][NTf2] & 368.15 & 0.938 & 0.639 & 31.9 \\
\hline
\end{tabular}




\begin{tabular}{|c|c|c|c|c|c|}
\hline 37 & [COC2C1morp][eFAP] & 318.15 & 0.774 & 0.502 & 35.1 \\
\hline 37 & [COC2C1morp][eFAP] & 328.15 & 0.811 & 0.498 & 38.6 \\
\hline 37 & {$[\mathrm{COC} 2 \mathrm{C} 1 \mathrm{morp}][\mathrm{eFAP}]$} & 338.15 & 0.849 & 0.494 & 41.8 \\
\hline 37 & {$[\mathrm{COC} 2 \mathrm{C} 1 \mathrm{morp}][\mathrm{eFAP}]$} & 348.15 & 0.888 & 0.491 & 44.7 \\
\hline 37 & [COC2C1morp][eFAP] & 358.15 & 0.926 & 0.488 & 47.3 \\
\hline 37 & {$[\mathrm{COC} 2 \mathrm{C} 1 \mathrm{morp}][\mathrm{eFAP}]$} & 368.15 & 0.965 & 0.484 & 49.8 \\
\hline 38 & {$[\mathrm{C} 4 \mathrm{C} 1 \mathrm{pyrr}][\mathrm{B}(\mathrm{CN}) 4]$} & 318.15 & 0.681 & 0.638 & 6.4 \\
\hline 38 & {$[\mathrm{C} 4 \mathrm{C} 1 \mathrm{pyrr}][\mathrm{B}(\mathrm{CN}) 4]$} & 323.15 & 0.688 & 0.633 & 8.0 \\
\hline 38 & {$[\mathrm{C} 4 \mathrm{C} 1 \mathrm{pyrr}][\mathrm{B}(\mathrm{CN}) 4]$} & 333.15 & 0.703 & 0.624 & 11.2 \\
\hline 38 & {$[\mathrm{C} 4 \mathrm{C} 1 \mathrm{pyrr}][\mathrm{B}(\mathrm{CN}) 4]$} & 343.15 & 0.718 & 0.616 & 14.2 \\
\hline 38 & {$[\mathrm{C} 4 \mathrm{C} 1 \mathrm{pyrr}][\mathrm{B}(\mathrm{CN}) 4]$} & 353.15 & 0.733 & 0.608 & 17.0 \\
\hline 38 & [C4C1pyrr][bob] & 333.15 & 1.13 & 0.880 & 22.1 \\
\hline 38 & [C4C1pyrr][bob] & 343.15 & 1.12 & 0.857 & 23.5 \\
\hline 38 & [C4C1pyrr] [bob] & 353.15 & 1.12 & 0.835 & 25.4 \\
\hline 38 & [C4C1pyrr] [bob] & 363.15 & 1.13 & 0.814 & 27.9 \\
\hline 38 & [C4C1pyrr][bob] & 373.15 & 1.13 & 0.795 & 29.7 \\
\hline 39 & [MTBDH][BETI] & 308.15 & 0.765 & 0.437 & 42.8 \\
\hline 39 & [MTBDH][BETI] & 318.15 & 0.776 & 0.440 & 43.3 \\
\hline 39 & {$[\mathrm{MTBDH}][\mathrm{BETI}]$} & 328.15 & 0.785 & 0.442 & 43.7 \\
\hline 39 & {$[\mathrm{MTBDH}][\mathrm{BETI}]$} & 338.15 & 0.795 & 0.444 & 44.2 \\
\hline 39 & {$[\mathrm{MTBDH}][\mathrm{BETI}]$} & 348.15 & 0.805 & 0.445 & 44.8 \\
\hline 40 & {$[\mathrm{C} 4 \mathrm{C} 1 \mathrm{pyrr}][\mathrm{C}(\mathrm{CN}) 3]$} & 318.15 & 0.709 & 0.785 & 10.7 \\
\hline 40 & {$[\mathrm{C} 4 \mathrm{C} 1 \mathrm{pyrr}][\mathrm{C}(\mathrm{CN}) 3]$} & 328.15 & 0.73 & 0.772 & 5.7 \\
\hline 40 & {$[\mathrm{C} 4 \mathrm{C} 1 \mathrm{pyrr}][\mathrm{C}(\mathrm{CN}) 3]$} & 338.15 & 0.752 & 0.759 & 1.0 \\
\hline 40 & {$[\mathrm{C} 4 \mathrm{C} 1 \mathrm{pyrr}][\mathrm{C}(\mathrm{CN}) 3]$} & 348.15 & 0.773 & 0.747 & 3.3 \\
\hline 40 & {$[\mathrm{C} 4 \mathrm{C} 1 \mathrm{pyrr}][\mathrm{C}(\mathrm{CN}) 3]$} & 358.15 & 0.794 & 0.736 & 7.3 \\
\hline 40 & {$[\mathrm{C} 4 \mathrm{C} 1 \mathrm{pyrr}][\mathrm{C}(\mathrm{CN}) 3]$} & 368.15 & 0.821 & 0.725 & 11.7 \\
\hline 41 & [C6-iQui][SCN] & 328.18 & 0.921 & 0.905 & 1.7 \\
\hline 41 & [C6-iQui] $[\mathrm{SCN}]$ & 338.18 & 0.943 & 0.886 & 6.1 \\
\hline 41 & [C6-iQui][SCN] & 348.18 & 0.963 & 0.867 & 10.0 \\
\hline 41 & [C6-iQui] $[\mathrm{SCN}]$ & 358.18 & 0.98 & 0.849 & 13.4 \\
\hline 41 & [C6-iQui][SCN] & 368.18 & 0.998 & 0.832 & 16.6 \\
\hline 42 & {$[\mathrm{COC} 2 \mathrm{C} 1 \mathrm{pip}][\mathrm{eFAP}]$} & 318.15 & 0.573 & 0.418 & 27.0 \\
\hline
\end{tabular}




\begin{tabular}{|c|c|c|c|c|c|}
\hline 42 & {$[\mathrm{COC} 2 \mathrm{C} 1 \mathrm{pip}][\mathrm{eFAP}]$} & 328.15 & 0.605 & 0.419 & 30.8 \\
\hline 42 & [COC2C1pip][eFAP] & 338.15 & 0.637 & 0.419 & 34.2 \\
\hline 42 & [COC2C1pip][eFAP] & 348.15 & 0.676 & 0.420 & 37.9 \\
\hline 42 & {$[\mathrm{COC} 2 \mathrm{C} 1 \mathrm{pip}][\mathrm{eFAP}]$} & 358.15 & 0.707 & 0.420 & 40.6 \\
\hline 42 & [COC2C1pip][eFAP] & 368.15 & 0.742 & 0.420 & 43.3 \\
\hline 43 & [COC2C1pyrr][eFAP] & 318.15 & 0.651 & 0.407 & 37.5 \\
\hline 43 & [COC2C1pyrr][eFAP $]$ & 328.15 & 0.687 & 0.408 & 40.6 \\
\hline 43 & {$[\mathrm{COC} 2 \mathrm{C} 1 \mathrm{pyrr}][\mathrm{eFAP}]$} & 338.15 & 0.722 & 0.410 & 43.3 \\
\hline 43 & [COC2C1pyrr][eFAP] & 348.15 & 0.76 & 0.411 & 45.9 \\
\hline 43 & [COC2C1pyrr][eFAP $]$ & 358.15 & 0.796 & 0.412 & 48.2 \\
\hline 43 & [COC2C1pyrr][eFAP] & 368.15 & 0.827 & 0.413 & 50.0 \\
\hline 44 & {$[\mathrm{HOC} 2 \mathrm{C} 1 \mathrm{im}][\mathrm{eFAP}]$} & 318.15 & 1.11 & 0.534 & 51.8 \\
\hline 44 & {$[\mathrm{HOC} 2 \mathrm{C} 1 \mathrm{im}][\mathrm{eFAP}]$} & 328.15 & 1.14 & 0.529 & 53.6 \\
\hline 44 & {$[\mathrm{HOC} 2 \mathrm{C} 1 \mathrm{im}][\mathrm{eFAP}]$} & 338.15 & 1.18 & 0.524 & 55.6 \\
\hline 44 & {$[\mathrm{HOC} 2 \mathrm{C} 1 \mathrm{im}][\mathrm{eFAP}]$} & 348.15 & 1.22 & 0.518 & 57.5 \\
\hline 44 & {$[\mathrm{HOC} 2 \mathrm{C} 1 \mathrm{im}][\mathrm{eFAP}]$} & 358.15 & 1.26 & 0.514 & 59.2 \\
\hline 44 & {$[\mathrm{HOC} 2 \mathrm{C} 1 \mathrm{im}][\mathrm{eFAP}]$} & 368.15 & 1.3 & 0.509 & 60.8 \\
\hline 45 & [C3C1pyrr][NTf2] & 323.15 & 0.86 & 0.647 & 24.8 \\
\hline 45 & [C3C1pyrr][NTf2] & 333.15 & 0.88 & 0.638 & 27.5 \\
\hline 45 & [C3C1pyrr][NTf2] & 343.15 & 0.89 & 0.630 & 29.2 \\
\hline 45 & [C4C1pyrr][NTf2] & 323.15 & 0.82 & 0.589 & 28.2 \\
\hline 45 & [C4C1pyrr][NTf2] & 333.15 & 0.83 & 0.583 & 29.8 \\
\hline 45 & [C4C1pyrr][NTf2] & 343.15 & 0.84 & 0.577 & 31.3 \\
\hline 46 & [C4C1im][PF6] & 313.15 & 1.34 & 1.227 & 8.5 \\
\hline 46 & [C4C1im][PF6] & 323.15 & 1.36 & 1.185 & 12.9 \\
\hline 46 & [C4C1im][PF6] & 333.15 & 1.38 & 1.146 & 17.0 \\
\hline 47 & {$[\mathrm{HOC} 2 \mathrm{C} 1 \mathrm{im}][\mathrm{eFAP}]$} & 318.15 & 1.071 & 0.534 & 50.1 \\
\hline 47 & {$[\mathrm{HOC} 2 \mathrm{C} 1 \mathrm{im}][\mathrm{eFAP}]$} & 323.15 & 1.074 & 0.532 & 50.5 \\
\hline 47 & {$[\mathrm{HOC} 2 \mathrm{C} 1 \mathrm{im}][\mathrm{eFAP}]$} & 333.15 & 1.077 & 0.526 & 51.1 \\
\hline 47 & {$[\mathrm{HOC} 2 \mathrm{C} 1 \mathrm{im}][\mathrm{eFAP}]$} & 343.15 & 1.08 & 0.521 & 51.8 \\
\hline 47 & {$[\mathrm{HOC} 2 \mathrm{C} 1 \mathrm{im}][\mathrm{eFAP}]$} & 353.15 & 1.085 & 0.516 & 52.4 \\
\hline 48 & [C5C1pip][NTf2] & 308.15 & 0.646 & 0.520 & 19.4 \\
\hline 48 & [C5C1pip][NTf2] & 318.15 & 0.665 & 0.517 & 22 \\
\hline
\end{tabular}




\begin{tabular}{|c|c|c|c|c|c|}
\hline 48 & [C5C1pip][NTf2] & 328.15 & 0.673 & 0.514 & 23.6 \\
\hline 48 & [C5C1pip][NTf2] & 338.15 & 0.69 & 0.511 & 25.9 \\
\hline 48 & [C5C1pip][NTf2] & 348.15 & 0.708 & 0.508 & 28.2 \\
\hline 48 & [C5C1pip][NTf2] & 358.15 & 0.722 & 0.505 & 30.0 \\
\hline 48 & [C6C1pip][NTf2] & 308.15 & 0.614 & 0.496 & 19.2 \\
\hline 48 & [C6C1pip][NTf2] & 318.15 & 0.627 & 0.494 & 21.2 \\
\hline 48 & [C6C1pip][NTf2] & 328.15 & 0.643 & 0.492 & 23.5 \\
\hline 48 & [C6C1pip][NTf2] & 338.15 & 0.655 & 0.489 & 25.3 \\
\hline 48 & [C6C1pip][NTf2] & 348.15 & 0.664 & 0.487 & 26.7 \\
\hline 48 & [C6C1pip][NTf2] & 358.15 & 0.675 & 0.485 & 28.2 \\
\hline 49 & {$[\mathrm{C} 4 \mathrm{C} 1 \mathrm{morp}][\mathrm{C}(\mathrm{CN}) 3]$} & 318.15 & 1.01 & 1.611 & 59.5 \\
\hline 49 & {$[\mathrm{C} 4 \mathrm{C} 1$ morp $][\mathrm{C}(\mathrm{CN}) 3]$} & 328.15 & 1.04 & 1.531 & 47.2 \\
\hline 49 & {$[\mathrm{C} 4 \mathrm{C} 1$ morp $][\mathrm{C}(\mathrm{CN}) 3]$} & 338.15 & 1.06 & 1.458 & 37.5 \\
\hline 49 & {$[\mathrm{C} 4 \mathrm{C} 1$ morp $][\mathrm{C}(\mathrm{CN}) 3]$} & 348.15 & 1.08 & 1.391 & 28.8 \\
\hline 49 & {$[\mathrm{C} 4 \mathrm{C} 1$ morp $][\mathrm{C}(\mathrm{CN}) 3]$} & 358.15 & 1.1 & 1.331 & 21.0 \\
\hline 49 & {$[\mathrm{C} 4 \mathrm{C} 1 \mathrm{morp}][\mathrm{C}(\mathrm{CN}) 3]$} & 368.15 & 1.12 & 1.276 & 14.0 \\
\hline 50 & {$[\mathrm{C} 4-4-\mathrm{C} 1 \mathrm{py}][\mathrm{N}(\mathrm{CN}) 2]$} & 308.15 & 0.822 & 1.319 & 60.5 \\
\hline 50 & {$[\mathrm{C} 4-4-\mathrm{C} 1 \mathrm{py}][\mathrm{N}(\mathrm{CN}) 2]$} & 318.15 & 0.844 & 1.278 & 51.4 \\
\hline 50 & {$[\mathrm{C} 4-4-\mathrm{C} 1 \mathrm{py}][\mathrm{N}(\mathrm{CN}) 2]$} & 328.15 & 0.868 & 1.239 & 42.7 \\
\hline 50 & {$[\mathrm{C} 4-4-\mathrm{C} 1 \mathrm{py}][\mathrm{N}(\mathrm{CN}) 2]$} & 338.15 & 0.89 & 1.202 & 35.0 \\
\hline 50 & {$[\mathrm{C} 4-4-\mathrm{C} 1 \mathrm{py}][\mathrm{N}(\mathrm{CN}) 2]$} & 348.15 & 0.911 & 1.167 & 28.1 \\
\hline 50 & {$[\mathrm{C} 4-4-\mathrm{C} 1 \mathrm{py}][\mathrm{N}(\mathrm{CN}) 2]$} & 358.15 & 0.937 & 1.134 & 21.0 \\
\hline 50 & {$[\mathrm{C} 4-4-\mathrm{C} 1 \mathrm{py}][\mathrm{N}(\mathrm{CN}) 2]$} & 368.15 & 0.955 & 1.103 & 15.5 \\
\hline 51 & [C4C1pip][NTf2] & 308.15 & 0.719 & 0.551 & 23.4 \\
\hline 51 & [C4C1pip][NTf2] & 318.15 & 0.724 & 0.547 & 24.4 \\
\hline 51 & [C4C1pip][NTf2] & 328.15 & 0.726 & 0.543 & 25.2 \\
\hline 51 & [C4C1pip][NTf2] & 338.15 & 0.733 & 0.539 & 26.5 \\
\hline 51 & [C4C1pip][NTf2] & 348.15 & 0.738 & 0.535 & 27.5 \\
\hline 51 & [C4C1pip][NTf2] & 358.15 & 0.753 & 0.531 & 29.4 \\
\hline 52 & {$[\mathrm{C} 16 \mathrm{C} 3 \mathrm{im}][\mathrm{BF} 4]$} & 323.15 & 0.82 & 0.568 & 30.7 \\
\hline 52 & {$[\mathrm{C} 16 \mathrm{C} 3 \mathrm{im}][\mathrm{BF} 4]$} & 333.15 & 0.75 & 0.560 & 25.4 \\
\hline 52 & {$[\mathrm{C} 16 \mathrm{C} 3 \mathrm{im}][\mathrm{BF} 4]$} & 343.15 & 0.71 & 0.552 & 22.3 \\
\hline \multicolumn{5}{|r|}{ AARD $\%$} & 24.1 \\
\hline
\end{tabular}




\section{Absolute Average Relative Deviation}

Absolute average relative deviation (AARD) is calculated according to the eq $3 \mathrm{~S}$, between the experimental and predicted data to verify the performance of COSMO-RS $\gamma_{s}^{\infty}$ prediction.

$$
A A R D / \%=\frac{1}{N} \sum\left|\frac{\gamma_{s, \exp }^{\infty}-\gamma_{s, C O S M O-R S}^{\infty}}{\gamma_{s, \exp }^{\infty}}\right| * 100
$$

Where $\gamma_{s, C O S M O-R S}^{\infty}$ and $\gamma_{s, \exp }^{\infty}$ are the predicted and experimental thiophene activity coefficients at infinite dilution in ILs, respectively. $\mathrm{N}$ indicates the number of available data. 


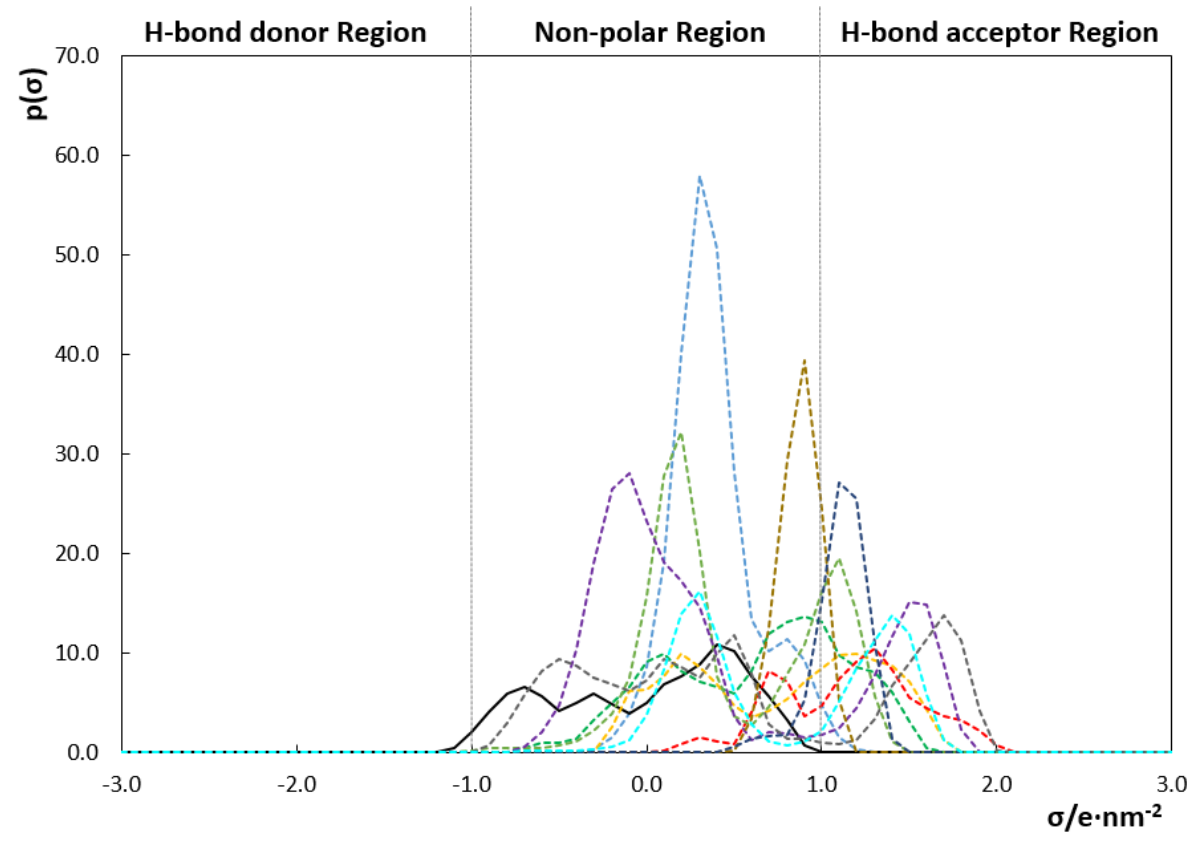

Figure S1. $\sigma$-profile of thiophene and anion computed by COSMO-RS. Symbols: (---), [eFAP]; (--), [B(CN)$\left.)_{4}\right] ;(--),\left[\mathrm{C}(\mathrm{CN})_{3}\right] ;(--),\left[\mathrm{OctSO}_{4}\right] ;(--),\left[\mathrm{NTf}_{2}\right] ;(---),\left[\mathrm{N}(\mathrm{CN})_{2}\right] ;(--),[\mathrm{BETI}] ;(--),[\mathrm{bob}] ;$ (---), [TOS]; (---),[CF $\left.\mathrm{CO}_{3}\right] ;(---),[\mathrm{SCN}] ;(---),\left[\mathrm{PF}_{6}\right] ;(---),\left[\mathrm{BF}_{4}\right] ;$ (-), thiophene. 


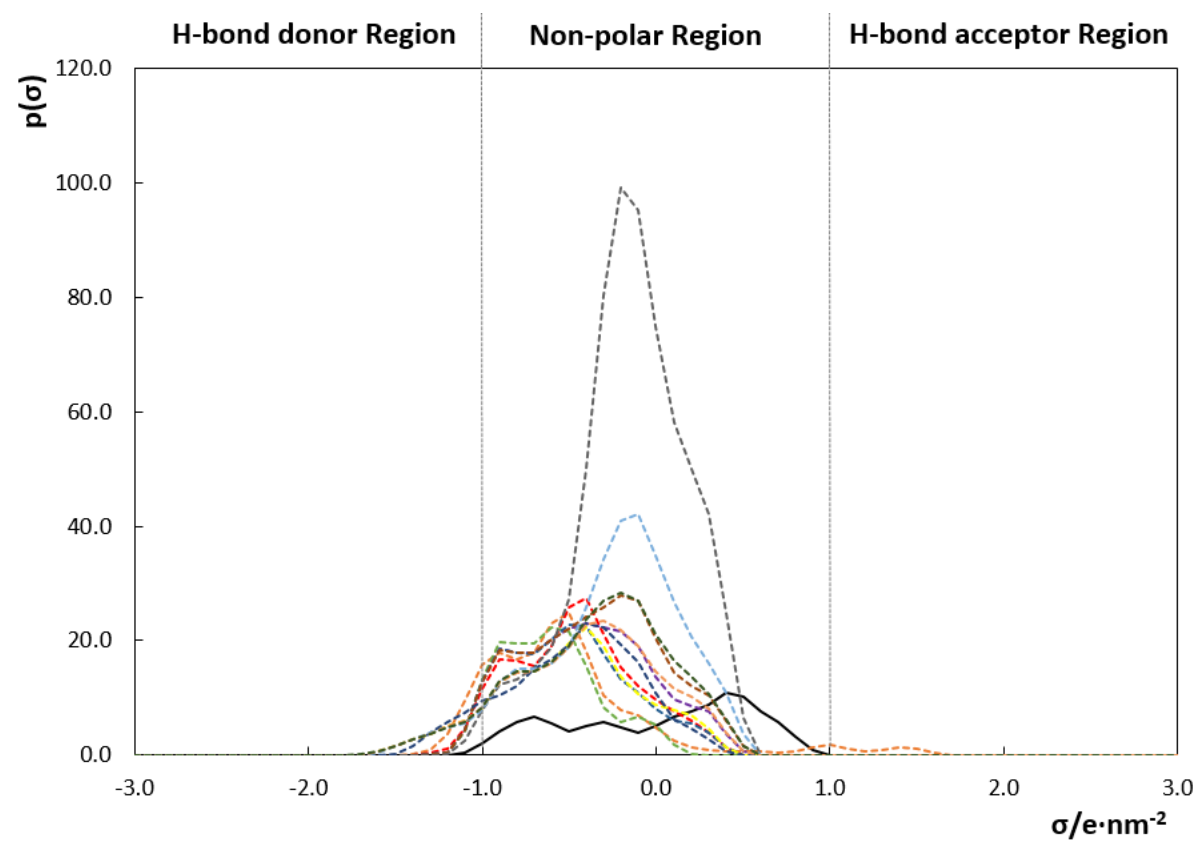

Figure S2. $\sigma$-profile of thiophene and cation computed by COSMO-RS. Symbols: (---),

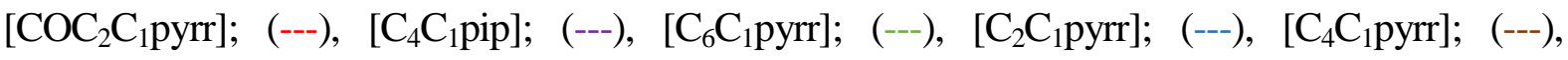

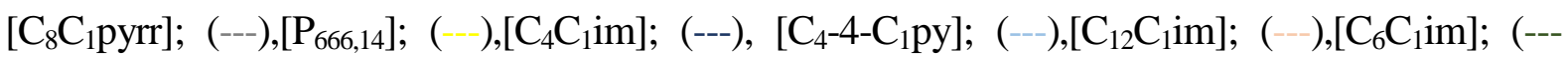
), $\left[\mathrm{C}_{8} \mathrm{C}_{1} \mathrm{im}\right] ;(-)$, thiophene. 


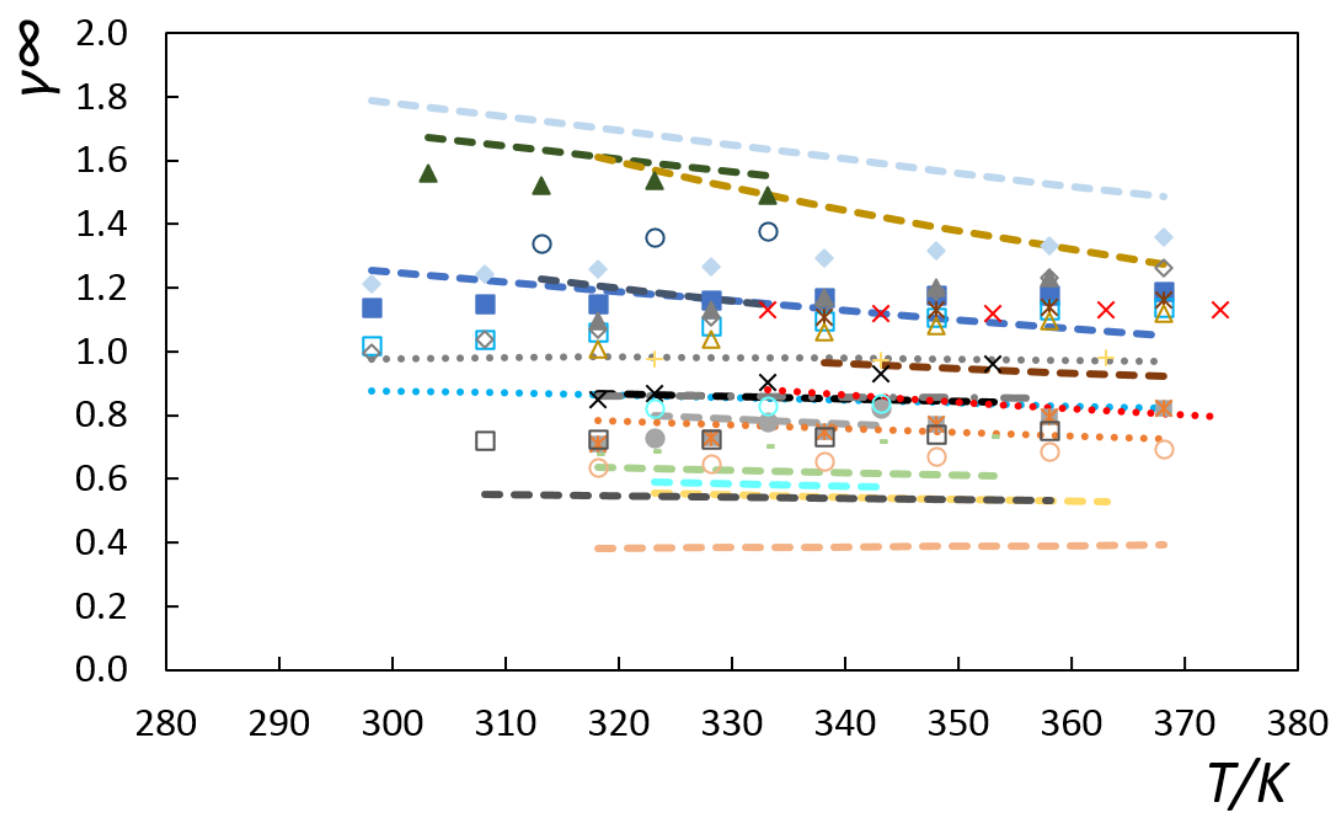

Figure S3. Thiophene activity coefficient at infinite dilutions as a function of temperature for $\mathrm{C}_{4} \mathrm{C}_{1} \mathrm{X}$ cation-based ILs. Symbols: $(\bullet,--),\left[\mathrm{C}_{4} \mathrm{C}_{1} \mathrm{im}\right]\left[\mathrm{OctSO}_{4}\right] ;^{1}(\square,--),\left[\mathrm{C}_{4} \mathrm{C}_{1} \mathrm{im}\right]\left[\mathrm{CF}_{3} \mathrm{SO}_{3}\right] ;^{3}(\diamond$, ),$\quad\left[\mathrm{C}_{4} \mathrm{C}_{1} \mathrm{im}\right][\mathrm{SCN}] ;^{6} \quad\left(\square,,^{--}\right), \quad\left[\mathrm{C}_{4} \mathrm{C}_{1}\right.$ pyrr $]\left[\mathrm{CF}_{3} \mathrm{SO}_{3}\right] ;^{10} \quad(\boldsymbol{\Delta},--), \quad\left[\mathrm{C}_{4} \mathrm{C}_{1} \mathrm{im}\right]\left[\mathrm{BF}_{4}\right] ;^{11} \quad(\diamond, \ldots)$, $\left[\mathrm{C}_{4} \mathrm{C}_{1}\right.$ pyrr $][\mathrm{SCN}] ;^{13} \quad(*,--), \quad\left[\mathrm{C}_{4} \mathrm{C}_{1} \mathrm{im}\right][\mathrm{TOS}] ;^{14} \quad(\mathrm{x},--), \quad\left[\mathrm{C}_{4} \mathrm{C}_{1}\right.$ pyrr $]\left[\mathrm{N}(\mathrm{CN})_{2}\right] ;^{.22} \quad(\triangle,-\cdot-)$, $\left[\mathrm{C}_{4} \mathrm{C}_{1}\right.$ pip $][\mathrm{SCN}] ;^{24} \quad(,--), \quad\left[\mathrm{C}_{4} \mathrm{C}_{1}\right.$ pyrr $]\left[\mathrm{B}(\mathrm{CN})_{4}\right] ;{ }^{26} \quad(+,-), \quad\left[\mathrm{C}_{4} \mathrm{C}_{1}\right.$ im $][\mathrm{BETI}] ;^{29} \quad(\circ,--)$, $\left[\mathrm{C}_{4} \mathrm{C}_{1}\right.$ pyrr $][\mathrm{eFAP}] ;^{32} \quad(\mathrm{x},-=), \quad\left[\mathrm{C}_{4} \mathrm{C}_{1}\right.$ pyrr $][\mathrm{bob}] ;^{38} \quad(*, \ldots), \quad\left[\mathrm{C}_{4} \mathrm{C}_{1}\right.$ pyrr $]\left[\mathrm{C}(\mathrm{CN})_{3}\right] ;^{40} \quad(\circ,--)$, $\left[\mathrm{C}_{4} \mathrm{C}_{1}\right.$ pyrr $]\left[\mathrm{NTf}_{2}\right] ;^{45} \quad(\circ,--), \quad\left[\mathrm{C}_{4} \mathrm{C}_{1} \mathrm{im}\right]\left[\mathrm{PF}_{6}\right] ;^{46} \quad(\triangle,-), \quad\left[\mathrm{C}_{4} \mathrm{C}_{1} \operatorname{morp}\right]\left[\mathrm{C}(\mathrm{CN})_{3}\right] ;^{49} \quad(\square,--)$, $\left[\mathrm{C}_{4} \mathrm{C}_{1} \operatorname{pip}\right]\left[\mathrm{NTf}_{2}\right] .^{51}$ 


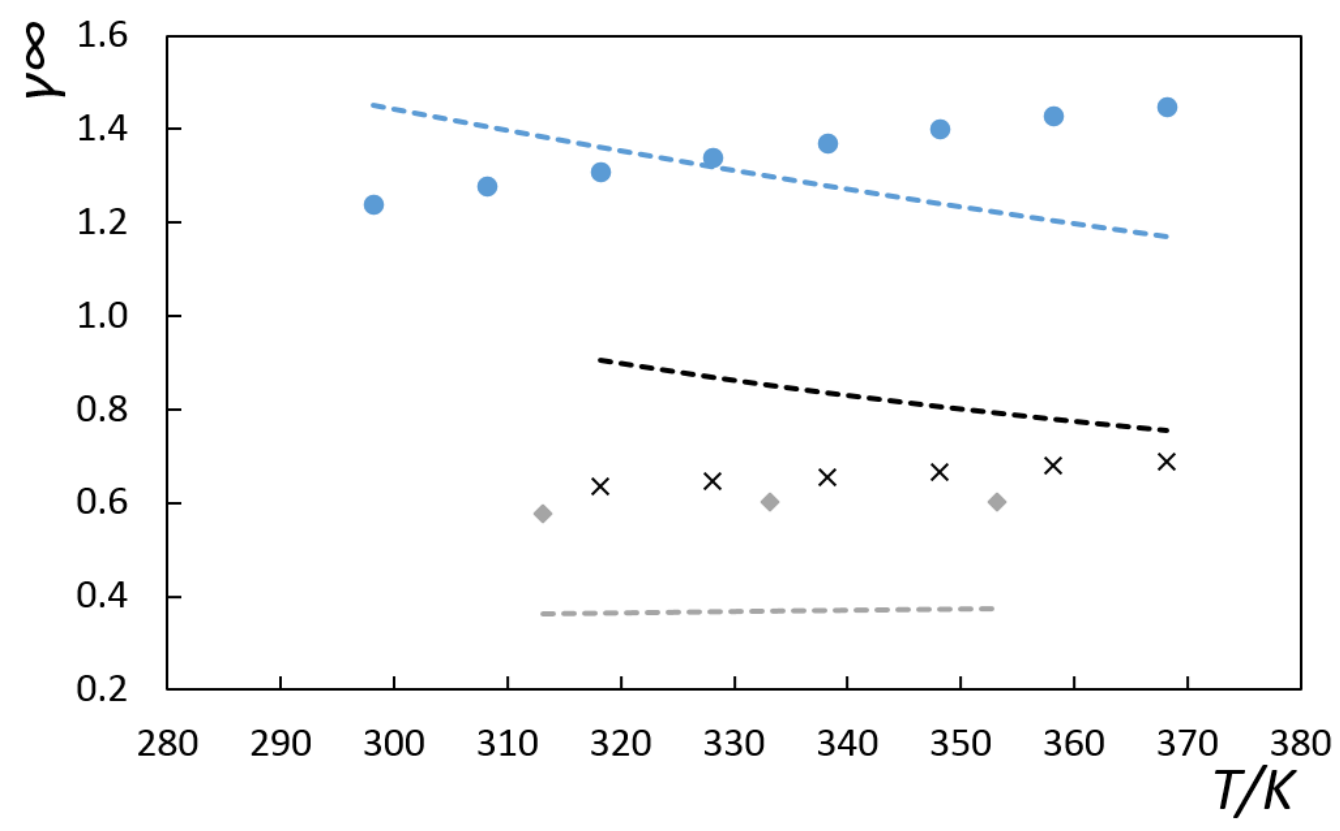

Figure S4. Thiophene activity coefficient at infinite dilutions as a function of temperature for $\mathrm{C}_{6} \mathrm{C}_{1}$ im cation-based ILs. Symbols: $(\bullet,--),\left[\mathrm{C}_{6} \mathrm{C}_{1} \mathrm{im}\right][\mathrm{SCN}] ;^{16}(\diamond,--),\left[\mathrm{C}_{6} \mathrm{C}_{1} \mathrm{im}\right][\mathrm{eFAP}] ;^{29}(\mathrm{x},--)$, $\left[\mathrm{C}_{6} \mathrm{C}_{1} \mathrm{im}\right][\mathrm{B}(\mathrm{CN}) 4] .{ }^{33}$ 


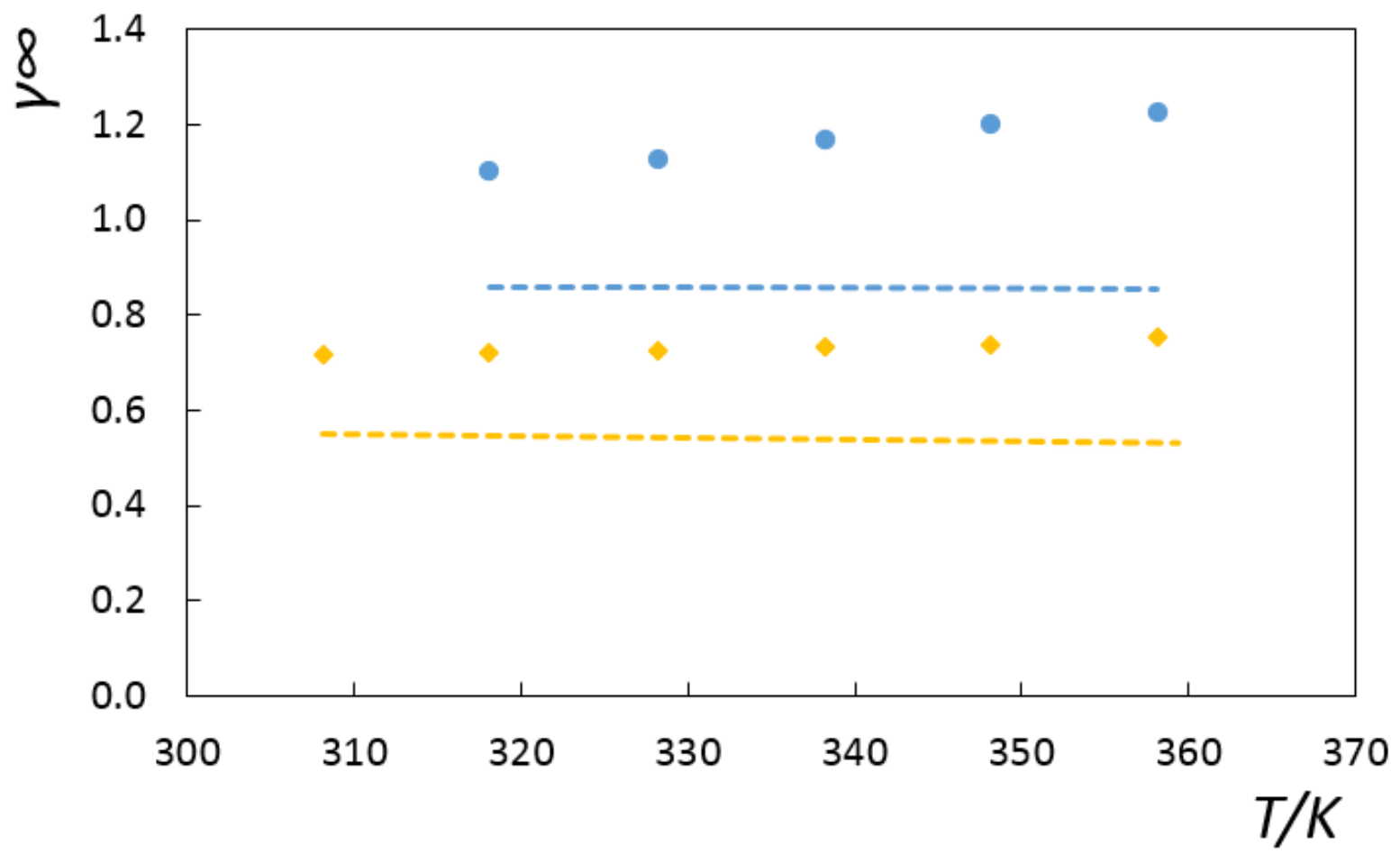

Figure S5. Thiophene activity coefficient at infinite dilutions as a function of temperature for $\mathrm{C}_{4} \mathrm{C}_{1}$ pip cation-based ILs. Symbols: $(\bullet,--),\left[\mathrm{C}_{4} \mathrm{C}_{1}\right.$ pip $][\mathrm{SCN}]{ }^{.4}(\diamond,--),\left[\mathrm{C}_{4} \mathrm{C}_{1}\right.$ pip $]\left[\mathrm{NTf}_{2}\right] .{ }^{51}$ 


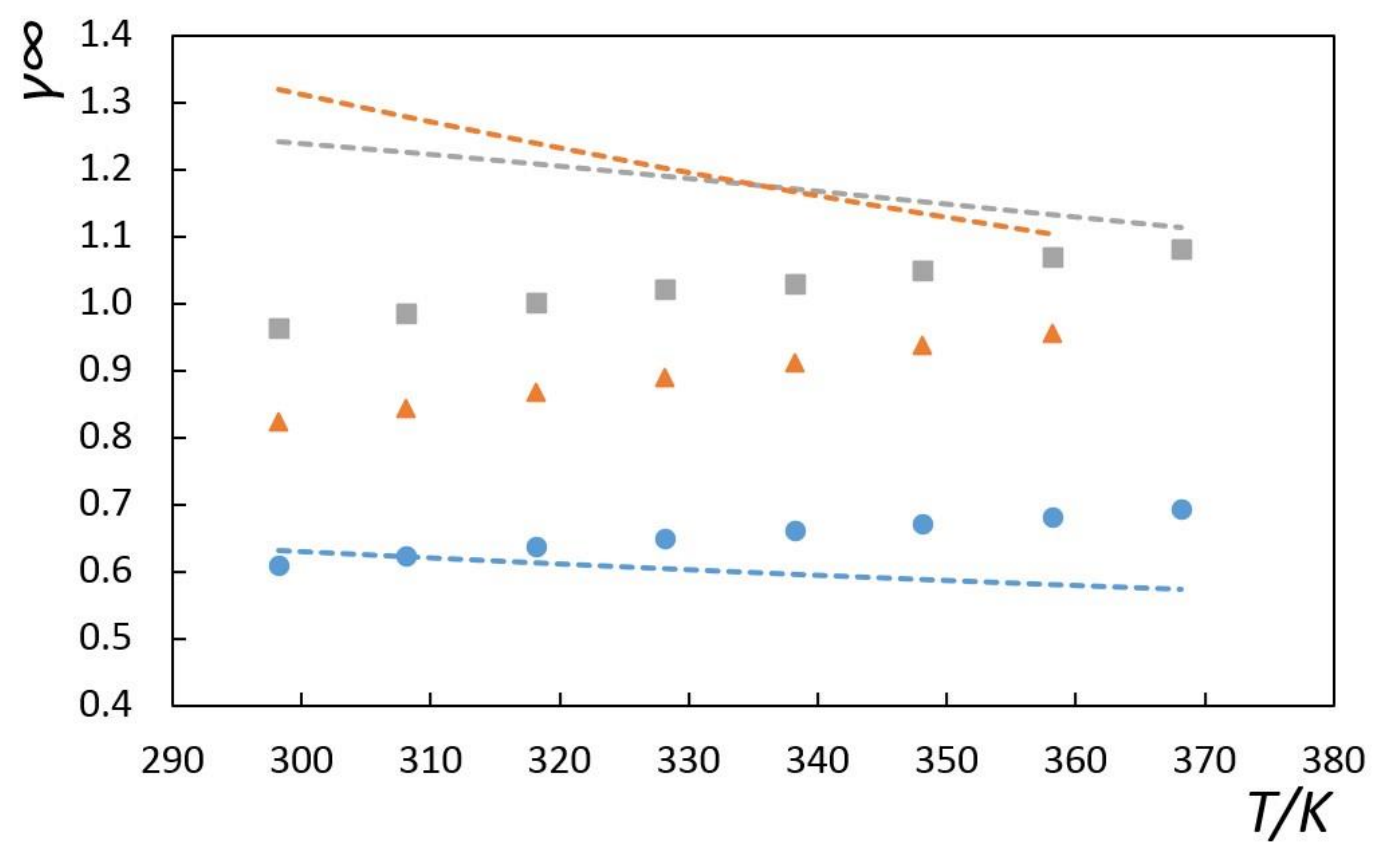

Figure S6. Thiophene activity coefficient at infinite dilutions as a function of temperature for $\mathrm{C}_{4}-4-\mathrm{C}_{1}$ py cation-based ILs. Symbols: (๑,--), $\left[\mathrm{C}_{4}-4-\mathrm{C}_{1}\right.$ py $]\left[\mathrm{NTf}_{2}\right] ;^{8} \quad(\triangle,--), \quad\left[\mathrm{C}_{4}-4-\right.$ $\mathrm{C}_{1}$ py $]\left[\mathrm{N}(\mathrm{CN})_{2}\right] ;{ }^{50}(\square,--),\left[\mathrm{C}_{4}-4-\mathrm{C}_{1}\right.$ py $][\mathrm{SCN}] .{ }^{13}$ 


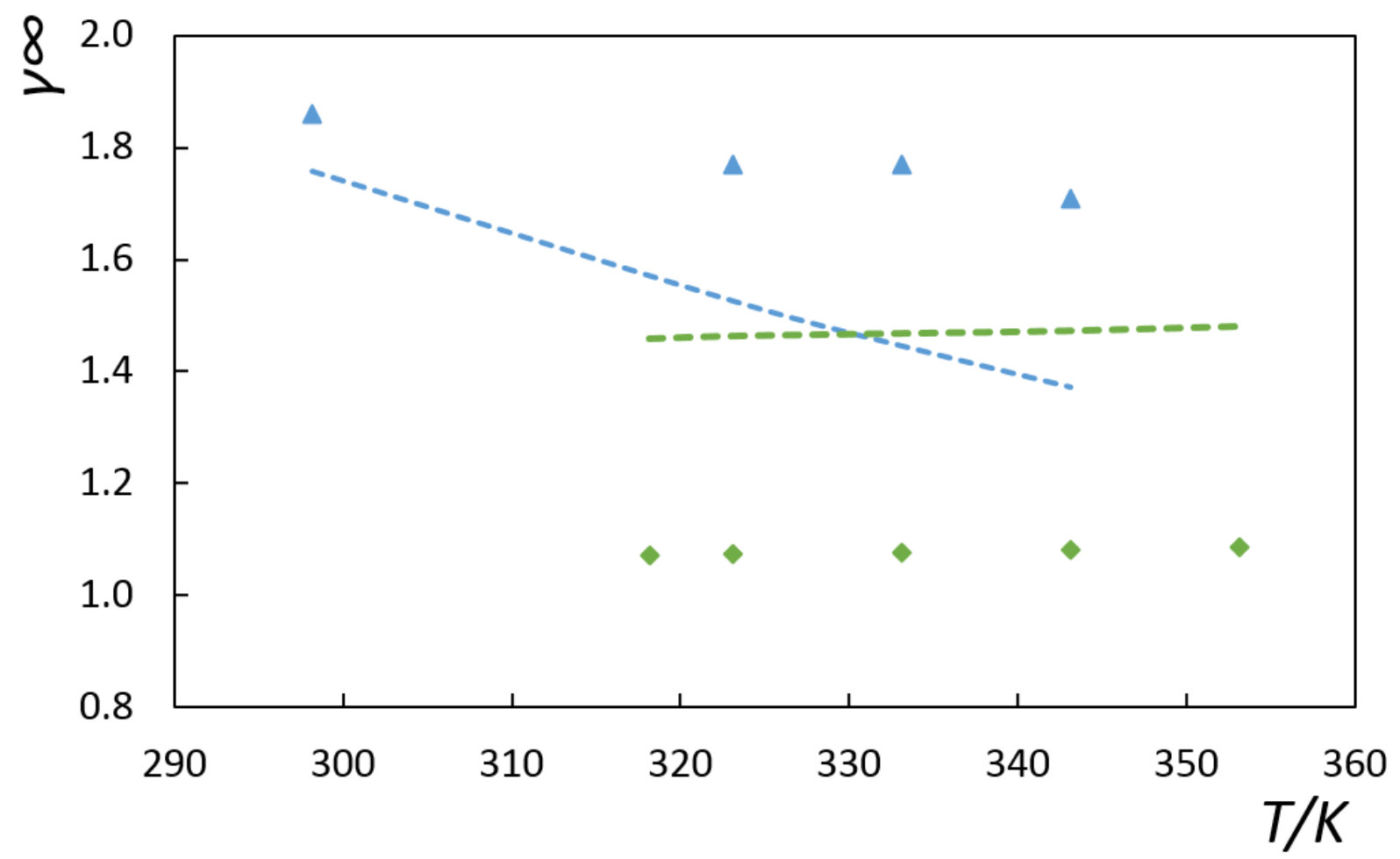

Figure S7. Thiophene activity coefficient at infinite dilutions as a function of temperature for

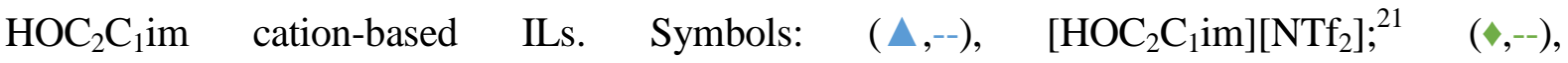
$\left[\mathrm{HOC}_{2} \mathrm{C}_{1} \mathrm{im}\right][\mathrm{eFAP}]{ }^{44}$ 


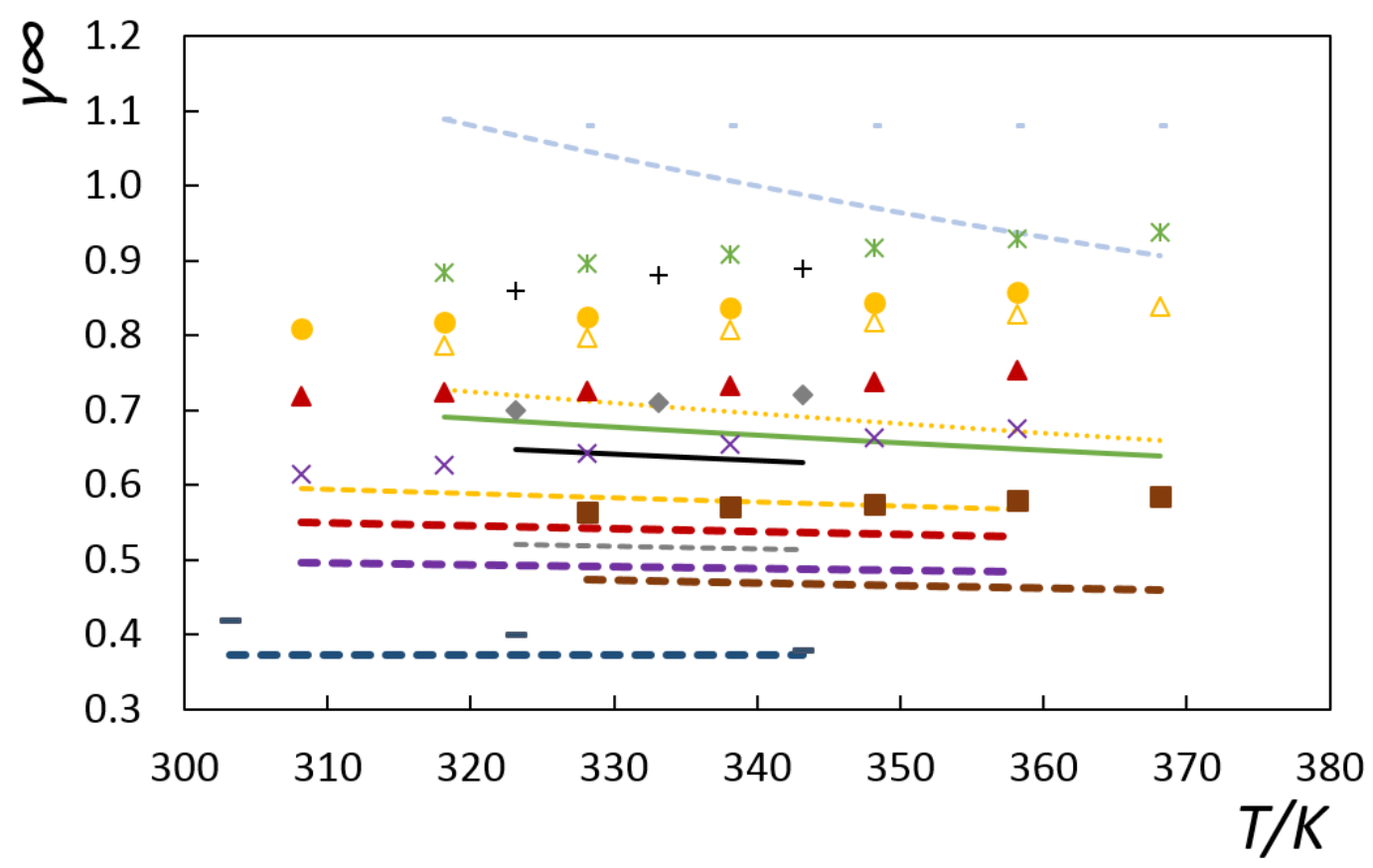

Figure S8. Thiophene activity coefficient at infinite dilutions as a function of temperature for NTf $f_{2}$ anion-based ILs. Symbols: $\left({ }^{*},-\right),\left[\mathrm{COC}_{2} \mathrm{C}_{1}\right.$ pyrr $]\left[\mathrm{NTf}_{2}\right]{ }^{36}(\mathrm{X},--),\left[\mathrm{C}_{6} \mathrm{C}_{1}\right.$ pip $]\left[\mathrm{NTf}_{2}\right]{ }^{48}(\boldsymbol{\Lambda},--)$, $\left[\mathrm{C}_{4} \mathrm{C}_{1}\right.$ pip $]\left[\mathrm{NTf}_{2}\right] ;{ }^{51} \quad(\bullet,-), \quad\left[\mathrm{C}_{3} \mathrm{C}_{1}\right.$ pip $]\left[\mathrm{NTf}_{2}\right] ;{ }^{18} \quad(+,-), \quad\left[\mathrm{C}_{3} \mathrm{C}_{1}\right.$ pyrr $]\left[\mathrm{NTf}_{2}\right] ;^{45} \quad(\diamond,--)$, $\left[\mathrm{C}_{6} \mathrm{C}_{1}\right.$ pyrr $]\left[\mathrm{NTf}_{2}\right] ;^{29} \quad(-,-), \quad[\mathrm{P} 14,6,6,6]\left[\mathrm{NTf}_{2}\right] ;^{5} \quad(\varpi,--),\left[\mathrm{C}_{8}\right.$-iQui $]\left[\mathrm{NTf}_{2}\right] ;^{27} \quad(,--)$, $\left.\left[\mathrm{COC}_{2} \mathrm{C}_{1} \operatorname{morp}\right]\left[\mathrm{NTf}_{2}\right]\right]^{35}(\triangle, \ldots),\left[\mathrm{COC}_{2} \mathrm{C}_{1}\right.$ pip $]\left[\mathrm{NTf}_{2}\right] .{ }^{34}$ 


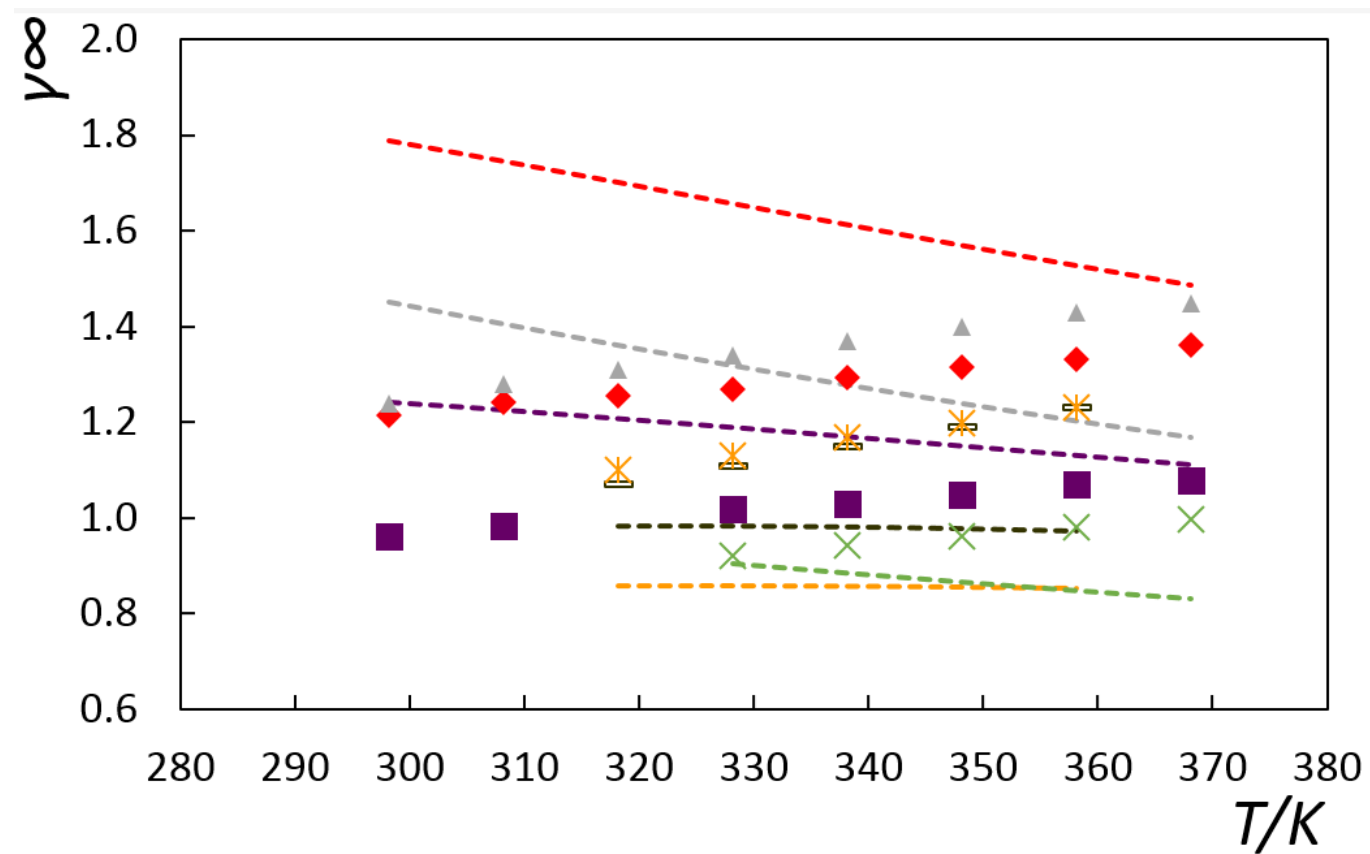

Figure S9. Thiophene activity coefficient at infinite dilutions as a function of temperature for SCN anion-based ILs. Symbols: (*,-), $\left[\mathrm{C}_{4} \mathrm{C}_{1} \operatorname{pip}\right][\mathrm{SCN}] ;^{24}(\diamond,--),\left[\mathrm{C}_{4} \mathrm{C}_{1} \mathrm{im}\right][\mathrm{SCN}] ;^{6}(\square,--),\left[\mathrm{C}_{4^{-}}\right.$ 4- $\mathrm{C}_{1}$ py $][\mathrm{SCN}] ;^{13}(\mathrm{X},--),\left[\mathrm{C}_{6}\right.$-iQui $][\mathrm{SCN}]{ }^{41}(\Delta,--),\left[\mathrm{C}_{6} \mathrm{C}_{1} \mathrm{im}\right][\mathrm{SCN}] ;^{16}(-,--),\left[\mathrm{C}_{4} \mathrm{C}_{1}\right.$ pyrr $][\mathrm{SCN}] .{ }^{13}$ 


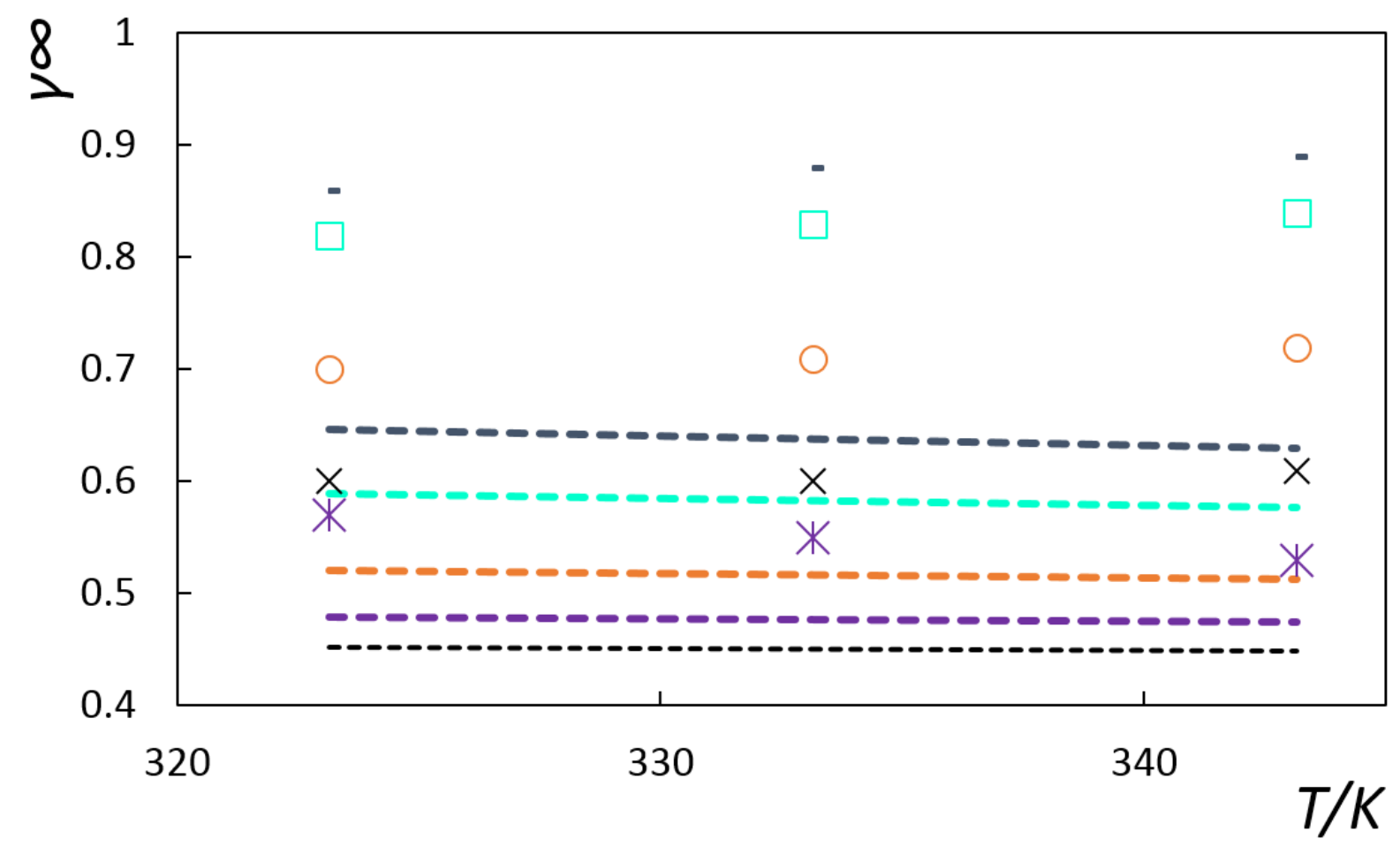

Figure S10. Thiophene activity coefficient at infinite dilutions as a function of temperature for $\mathrm{NTf}_{2}$ anion-based ILs. Symbols: (*,--), $\left[\mathrm{C}_{8} \mathrm{C}_{1}\right.$ pyrr $]\left[\mathrm{NTf}_{2}\right]{ }^{30}(\circ,--),\left[\mathrm{C}_{6} \mathrm{C}_{1}\right.$ pyrr $]\left[\mathrm{NTf}_{2}\right] ;^{30}$ $(\square,--),\left[\mathrm{C}_{4} \mathrm{C}_{1}\right.$ pyrr $\left.]\left[\mathrm{NTf}_{2}\right]\right]^{45} \quad(-,--),\left[\mathrm{C}_{3} \mathrm{C}_{1}\right.$ pyrr $\left.]\left[\mathrm{NTf}_{2}\right]\right]^{45}(\mathrm{x},--),\left[\mathrm{C}_{10} \mathrm{C}_{1}\right.$ pyrr $]\left[\mathrm{NTf}_{2}\right] .{ }^{30}$ 


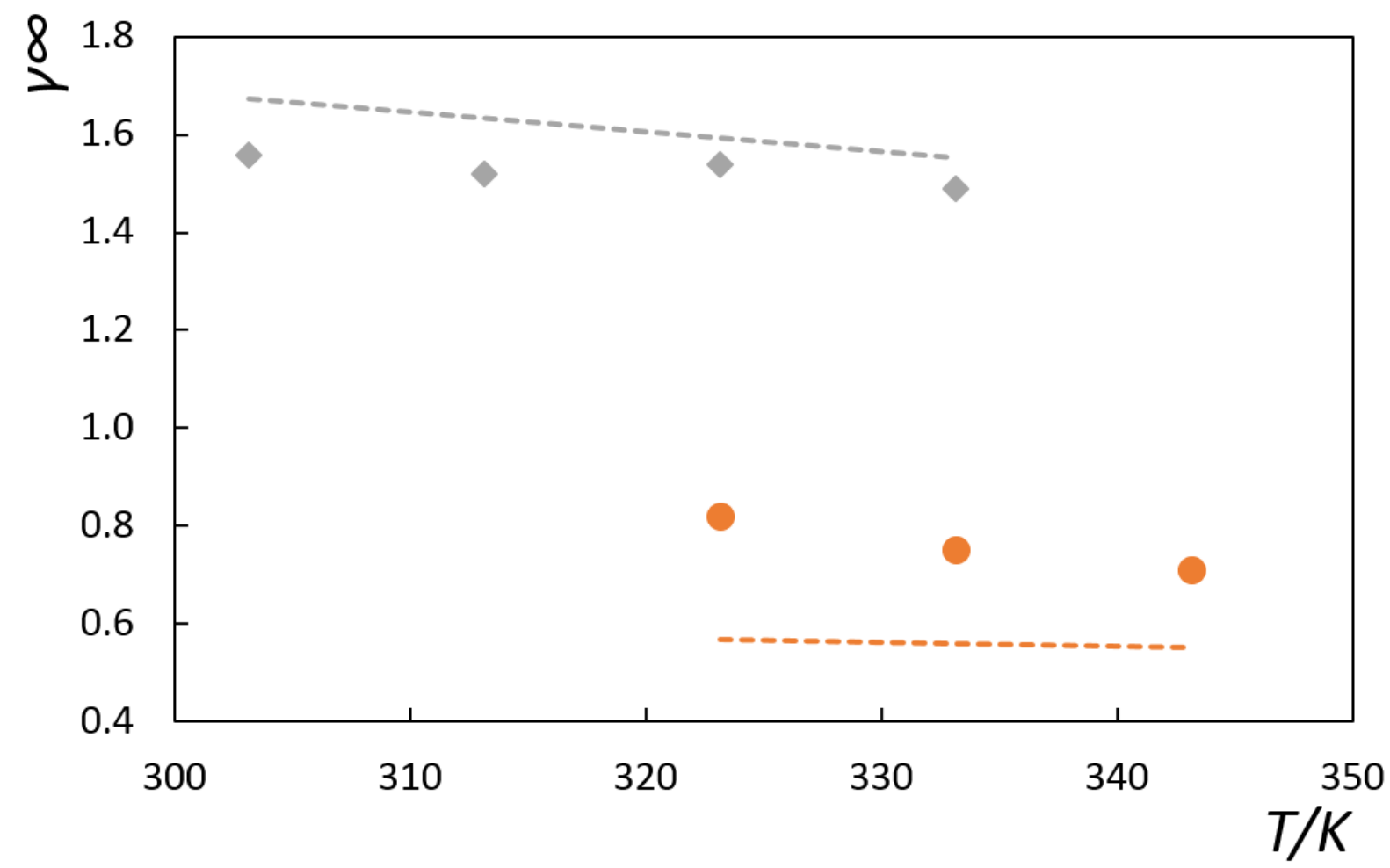

Figure S11. Thiophene activity coefficient at infinite dilutions as a function of temperature for $\mathrm{BF}_{4}$ anion-based ILs. Symbols: $(\bullet,--),\left[\mathrm{C}_{16} \mathrm{C}_{3} \mathrm{im}\right]\left[\mathrm{BF}_{4}\right] ;{ }^{52}(\diamond,--),\left[\mathrm{C}_{4} \mathrm{C}_{1} \mathrm{im}\right]\left[\mathrm{BF}_{4}\right] .{ }^{11}$ 


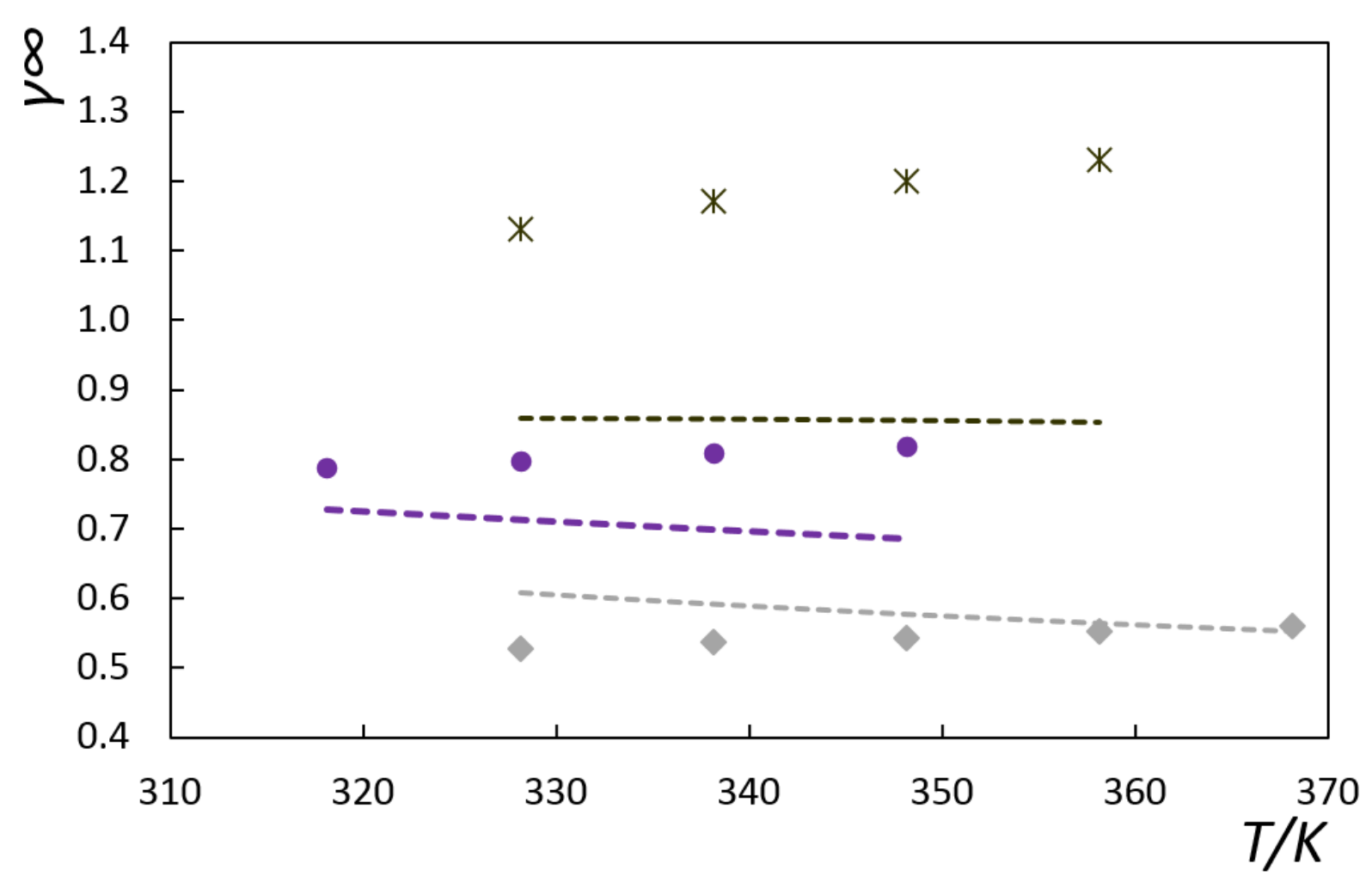

Figure S12. Thiophene activity coefficient at infinite dilutions as a function of temperature for $\mathrm{B}(\mathrm{CN})_{4}$ anion-based ILs. Symbols: $(\bullet,--), \quad\left[\mathrm{C}_{6} \mathrm{C}_{1} \mathrm{im}\right]\left[\mathrm{B}(\mathrm{CN})_{4}\right] ;{ }^{33} \quad(\diamond,--)$, $\left[\mathrm{C}_{10} \mathrm{C}_{1} \mathrm{im}\right]\left[\mathrm{B}(\mathrm{CN})_{4}\right] .^{15}\left({ }^{*},--\right),\left[\mathrm{C}_{2} \mathrm{C}_{1} \mathrm{im}\right]\left[\mathrm{B}(\mathrm{CN})_{4}\right] .^{23}$ 


\section{References}

1. Mutelet, F.; Jaubert, J.-N., Accurate measurements of thermodynamic properties of solutes in ionic liquids using inverse gas chromatography. Journal of Chromatography A 2006, 1102, (1), 256-267.

2. Mutelet, F.; Jaubert, J.-N.; Rogalski, M.; Boukherissa, M.; Dicko, A., Thermodynamic properties of mixtures containing ionic liquids: Activity coefficients at infinite dilution of organic compounds in 1-propyl boronic acid-3-alkylimidazolium bromide and 1-propenyl-3-alkylimidazolium bromide using inverse gas chromatography. Journal of Chemical \& Engineering Data 2006, 51, (4), 1274-1279.

3. Domanska, U.; Marciniak, A., Activity coefficients at infinite dilution measurements for organic solutes and water in the ionic liquid 1-butyl-3-methylimidazolium trifluoromethanesulfonate. The Journal of Physical Chemistry B 2008, 112, (35), 1110011105.

4. Mutelet, F.; Jaubert, J.-N.; Rogalski, M.; Harmand, J.; Sindt, M.; Mieloszynski, J.-L., Activity coefficients at infinite dilution of organic compounds in 1-(meth) acryloyloxyalkyl3-methylimidazolium bromide using inverse gas chromatography. The Journal of Physical Chemistry B 2008, 112, (12), 3773-3785.

5. Revelli, A.-L.; Sprunger, L. M.; Gibbs, J.; Acree Jr, W. E.; Baker, G. A.; Mutelet, F., Activity coefficients at infinite dilution of organic compounds in trihexyl (tetradecyl) phosphonium bis (trifluoromethylsulfonyl) imide using inverse gas chromatography. Journal of Chemical \& Engineering Data 2009, 54, (3), 977-985.

6. Domańska, U.; Laskowska, M., Measurements of activity coefficients at infinite dilution of aliphatic and aromatic hydrocarbons, alcohols, thiophene, tetrahydrofuran, MTBE, and water in ionic liquid [BMIM][SCN] using GLC. The Journal of Chemical Thermodynamics 2009, 41, (5), 645-650.

7. Domańska, U.; Marciniak, A., Activity coefficients at infinite dilution measurements for organic solutes and water in the ionic liquid triethylsulphonium bis (trifluoromethylsulfonyl) imide. The Journal of Chemical Thermodynamics 2009, 41, (6), 754-758.

8. Domańska, U.; Marciniak, A., Activity coefficients at infinite dilution measurements for organic solutes and water in the ionic liquid 4-methyl-N-butyl-pyridinium bis 
(trifluoromethylsulfonyl)-imide. The Journal of Chemical Thermodynamics 2009, 41, (12), $1350-1355$.

9. Domańska, U.; Marciniak, A., Activity coefficients at infinite dilution measurements for organic solutes and water in the 1-hexyloxymethyl-3-methyl-imidazolium and 1, 3dihexyloxymethyl-imidazolium bis (trifluoromethylsulfonyl)-imide ionic liquids - The cation influence. Fluid Phase Equilibria 2009, 286, (2), 154-161.

10. Domańska, U.; Redhi, G. G.; Marciniak, A., Activity coefficients at infinite dilution measurements for organic solutes and water in the ionic liquid 1-butyl-1-methylpyrrolidinium trifluoromethanesulfonate using GLC. Fluid phase equilibria 2009, 278, (1), 97-102.

11. Revelli, A.-L.; Mutelet, F.; Turmine, M.; Solimando, R.; Jaubert, J.-N. 1., Activity coefficients at infinite dilution of organic compounds in 1-butyl-3-methylimidazolium tetrafluoroborate using inverse gas chromatography. Journal of Chemical \& Engineering Data 2008, 54, (1), 90-101.

12. Blahut, A.; Sobota, M.; Dohnal, V.; Vrbka, P., Activity coefficients at infinite dilution of organic solutes in the ionic liquid 1-ethyl-3-methylimidazolium methanesulfonate. Fluid Phase Equilibria 2010, 299, (2), 198-206.

13. Domańska, U.; Królikowska, M., Measurements of activity coefficients at infinite dilution in solvent mixtures with thiocyanate-based ionic liquids using GLC technique. The Journal of Physical Chemistry B 2010, 114, (25), 8460-8466.

14. Domańska, U.; Królikowski, M., Determination of Activity Coefficients at Infinite Dilution of 35 Solutes in the Ionic Liquid, 1-Butyl-3-methylimidazolium Tosylate, Using Gas- Liquid Chromatography. Journal of Chemical \& Engineering Data 2010, 55, (11), 4817-4822.

15. Domanska, U.; Marciniak, A., Physicochemical properties and activity coefficients at infinite dilution for organic solutes and water in the ionic liquid 1-decyl-3methylimidazolium tetracyanoborate. The Journal of Physical Chemistry B 2010, 114, (49), 16542-16547.

16. Domańska, U.; Marciniak, A.; Królikowska, M.; Arasimowicz, M., Activity coefficients at infinite dilution measurements for organic solutes and water in the ionic liquid 1-hexyl-3-methylimidazolium thiocyanate. Journal of Chemical \& Engineering Data 2010, $55,(7), 2532-2536$.

17. Domańska, U.; Paduszyński, K., Gas-liquid chromatography measurements of activity coefficients at infinite dilution of various organic solutes and water in tri-iso- 
butylmethylphosphonium tosylate ionic liquid. The Journal of Chemical Thermodynamics 2010, 42, (6), 707-711.

18. Domańska, U.; Paduszyński, K., Measurements of activity coefficients at infinite dilution of organic solutes and water in 1-propyl-1-methylpiperidinium bis (trifluoromethyl) sulfonyl \} imide ionic liquid using glc. The Journal of Chemical Thermodynamics 2010, 42, (11), 1361-1366.

19. Marciniak, A.; Wlazlo, M., Activity coefficients at infinite dilution measurements for organic solutes and water in the ionic liquid 1-butyl-3-methyl-pyridinium trifluoromethanesulfonate. Journal of Chemical \& Engineering Data 2010, 55, (9), 32083211.

20. Marciniak, A.; Wlazło, M., Activity coefficients at infinite dilution measurements for organic solutes and water in the ionic liquid 1-(3-hydroxypropyl) pyridinium trifluorotris (perfluoroethyl) phosphate. The Journal of Physical Chemistry B 2010, 114, (20), 6990-6994. 21. Revelli, A.-L.; Mutelet, F.; Jaubert, J.-N.; Garcia-Martinez, M.; Sprunger, L. M.; Acree Jr, W. E.; Baker, G. A., Study of ether-, alcohol-, or cyano-functionalized ionic liquids using inverse gas chromatography. Journal of Chemical \& Engineering Data 2010, 55, (7), 2434-2443.

22. Blahut, A.; Dohnal, V., Interactions of volatile organic compounds with the ionic liquid 1-butyl-1-methylpyrrolidinium dicyanamide. Journal of Chemical \& Engineering Data 2011, 56, (12), 4909-4918.

23. Domańska, U.; Królikowska, M.; Acree, W. E.; Baker, G. A., Activity coefficients at infinite dilution measurements for organic solutes and water in the ionic liquid 1-ethyl-3methylimidazolium tetracyanoborate. The Journal of Chemical Thermodynamics 2011, 43, (7), 1050-1057.

24. Domańska, U.; Królikowska, M., Measurements of activity coefficients at infinite dilution for organic solutes and water in the ionic liquid 1-butyl-1-methylpiperidinium thiocyanate. Journal of Chemical \& Engineering Data 2010, 56, (1), 124-129.

25. Domańska, U.; Królikowski, M., Thermodynamics and activity coefficients at infinite dilution measurements for organic solutes and water in the ionic liquid N-hexyl-3methylpyridinium tosylate. The Journal of Physical Chemistry B 2011, 115, (22), 7397-7404.

26. Domańska, U.; Królikowski, M.; Acree, W. E., Thermodynamics and activity coefficients at infinite dilution measurements for organic solutes and water in the ionic liquid 1-butyl-1-methylpyrrolidinium tetracyanoborate. The Journal of Chemical Thermodynamics 2011, 43, (12), 1810-1817. 
27. Domańska, U.; Zawadzki, M.; Królikowska, M.; Tshibangu, M. M.; Ramjugernath, D.; Letcher, T. M., Measurements of activity coefficients at infinite dilution of organic compounds and water in isoquinolinium-based ionic liquid [C 8 iQuin][NTf 2] using GLC. The Journal of Chemical Thermodynamics 2011, 43, (3), 499-504.

28. Marciniak, A., Activity coefficients at infinite dilution and physicochemical properties for organic solutes and water in the ionic liquid 1-(3-hydroxypropyl) pyridinium bis (trifluoromethylsulfonyl)-amide. The Journal of Chemical Thermodynamics 2011, 43, (10), 1446-1452.

29. Moïse, J.-C.; Mutelet, F.; Jaubert, J.-N.; Grubbs, L. M.; Acree Jr, W. E.; Baker, G. A., Activity coefficients at infinite dilution of organic compounds in four new imidazoliumbased ionic liquids. Journal of Chemical \& Engineering Data 2011, 56, (7), 3106-3114.

30. Acree Jr, W. E.; Baker, G. A.; Revelli, A.-L.; Moïse, J.-C.; Mutelet, F., Activity coefficients at infinite dilution for organic compounds dissolved in 1-alkyl-1methylpyrrolidinium bis (trifluoromethylsulfonyl) imide ionic liquids having six-, eight-, and ten-carbon alkyl chains. Journal of Chemical \& Engineering Data 2012, 57, (12), 3510-3518. 31. Domańska, U.; Królikowski, M., Measurements of activity coefficients at infinite dilution for organic solutes and water in the ionic liquid 1-ethyl-3-methylimidazolium methanesulfonate. The Journal of Chemical Thermodynamics 2012, 54, 20-27.

32. Domańska, U.; Lukoshko, E. V.; Królikowski, M., Measurements of activity coefficients at infinite dilution for organic solutes and water in the ionic liquid 1-butyl-1methylpyrrolidinium tris (pentafluoroethyl) trifluorophosphate ([BMPYR][FAP]). Chemical Engineering Journal 2012, 183, 261-270.

33. Domańska, U.; Lukoshko, E. V.; Wlazło, M., Measurements of activity coefficients at infinite dilution for organic solutes and water in the ionic liquid 1-hexyl-3methylimidazolium tetracyanoborate. The Journal of Chemical Thermodynamics 2012, 47, 389-396.

34. Marciniak, A.; Wlazło, M., Activity coefficients at infinite dilution and physicochemical properties for organic solutes and water in the ionic liquid 1-(2methoxyethyl)-1-methylpiperidinium bis (trifluoromethylsulfonyl)-amide. The Journal of Chemical Thermodynamics 2012, 49, 137-145.

35. Marciniak, A.; Wlazło, M., Activity coefficients at infinite dilution and physicochemical properties for organic solutes and water in the ionic liquid 4-(2methoxyethyl)-4-methylmorpholinium bis (trifluoromethylsulfonyl)-amide. The Journal of Chemical Thermodynamics 2012, 47, 382-388. 
36. Marciniak, A.; Wlazło, M., Activity coefficients at infinite dilution and physicochemical properties for organic solutes and water in the ionic liquid 1-(2methoxyethyl)-1-methylpyrrolidinium bis(trifluoromethylsulfonyl)-amide. The Journal of Chemical Thermodynamics 2012, 54, 90-96.

37. Wlazło, M.; Marciniak, A., Activity coefficients at infinite dilution and physicochemical properties for organic solutes and water in the ionic liquid 4-(2methoxyethyl)-4-methylmorpholinium trifluorotris (perfluoroethyl) phosphate. The Journal of Chemical Thermodynamics 2012, 54, 366-372.

38. Blahut, A.; Dohnal, V., Interactions of volatile organic compounds with the ionic liquids 1-butyl-1-methylpyrrolidinium tetracyanoborate and 1-butyl-1-methylpyrrolidinium bis (oxalato) borate. The Journal of Chemical Thermodynamics 2013, 57, 344-354.

39. Domańska, U.; Królikowski, M.; Acree, W. E.; Baker, G. A., Physicochemical properties and activity coefficients at infinite dilution for organic solutes and water in a novel bicyclic guanidinium superbase-derived protic ionic liquid. The Journal of Chemical Thermodynamics 2013, 58, 62-69.

40. Domańska, U.; Lukoshko, E. V., Measurements of activity coefficients at infinite dilution for organic solutes and water in the ionic liquid 1-butyl-1-methylpyrrolidinium tricyanomethanide. The Journal of Chemical Thermodynamics 2013, 66, 144-150.

41. Królikowska, M.; Karpińska, M.; Królikowski, M., Measurements of activity coefficients at infinite dilution for organic solutes and water in N-hexylisoquinolinium thiocyanate,[HiQuin][SCN] using GLC. The Journal of Chemical Thermodynamics 2013, 62, $1-7$.

42. Marciniak, A.; Wlazło, M., Activity coefficients at infinite dilution and physicochemical properties for organic solutes and water in the ionic liquid 1-(2methoxyethyl)-1-methylpiperidinium trifluorotris(perfluoroethyl)phosphate. The Journal of Chemical Thermodynamics 2013, 57, 197-202.

43. Marciniak, A.; Wlazło, M., Activity coefficients at infinite dilution and physicochemical properties for organic solutes and water in the ionic liquid 1-(2methoxyethyl)-1-methylpyrrolidinium trifluorotris (perfluoroethyl) phosphate. The Journal of Chemical Thermodynamics 2013, 60, 57-62.

44. Marciniak, A.; Wlazło, M., Activity coefficients at infinite dilution and physicochemical properties for organic solutes and water in the ionic liquid 1-(2hydroxyethyl)-3-methylimidazolium trifluorotris (perfluoroethyl) phosphate. The Journal of Chemical Thermodynamics 2013, 64, 114-119. 
45. Mutelet, F.; Hassan, E.-S. R.; Stephens, T. W.; Acree Jr, W. E.; Baker, G. A., Activity coefficients at infinite dilution for organic solutes dissolved in three 1-alkyl-1methylpyrrolidinium bis (trifluoromethylsulfonyl) imide ionic liquids bearing short linear alkyl side chains of three to five carbons. Journal of Chemical \& Engineering Data 2013, 58, (8), 2210-2218.

46. Xu, Q.; Su, B.; Luo, X.; Xing, H.; Bao, Z.; Yang, Q.; Yang, Y.; Ren, Q., Accurate measurements of infinite dilution activity coefficients using gas chromatography with staticwall-coated open-tubular columns. Analytical chemistry 2012, 84, (21), 9109-9115.

47. Órfão, E. F.; Dohnal, V.; Blahut, A., Infinite dilution activity coefficients of volatile organic compounds in two ionic liquids composed of the tris (pentafluoroethyl) trifluorophosphate ([FAP]) anion and a functionalized cation. The Journal of Chemical Thermodynamics 2013, 65, 53-64.

48. Paduszyński, K.; Domańska, U., Experimental and theoretical study on infinite dilution activity coefficients of various solutes in piperidinium ionic liquids. The Journal of Chemical Thermodynamics 2013, 60, 169-178.

49. Domańska, U.; Lukoshko, E. V., Thermodynamics and activity coefficients at infinite dilution for organic solutes and water in the ionic liquid 1-butyl-1-methylmorpholinium tricyanomethanide. The Journal of Chemical Thermodynamics 2014, 68, 53-59.

50. Królikowski, M.; Królikowska, M., The study of activity coefficients at infinite dilution for organic solutes and water in 1-butyl-4-methylpyridinium dicyanamide,[B 4 MPy][DCA] using GLC. The Journal of Chemical Thermodynamics 2014, 68, 138-144.

51. Paduszynski, K.; Domanska, U., Limiting activity coefficients and gas-liquid partition coefficients of various solutes in piperidinium ionic liquids: measurements and LSER calculations. The Journal of Physical Chemistry B 2011, 115, (25), 8207-8215.

52. Mutelet, F.; Jaubert, J.-N., Measurement of activity coefficients at infinite dilution in 1-hexadecyl-3-methylimidazolium tetrafluoroborate ionic liquid. The Journal of Chemical Thermodynamics 2007, 39, (8), 1144-1150. 